NISTIR 8003

\title{
Design and Testing of a Mobile Touchscreen Interface for Multi-Modal Biometric Capture
}

\author{
Kristen K. Greene
}

Ross J. Micheals

Kayee Kwong Gregory P. Fiumara

http://dx.doi.org/10.6028/NIST.IR.8003

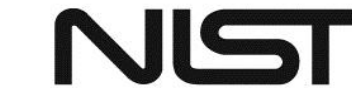

National Institute of Standards and Technology U.S. Department of Commerce 
NISTIR 8003

\title{
Design and Testing of a Mobile Touchscreen Interface for Multi-Modal Biometric Capture
}

\author{
Kristen K. Greene \\ Ross J. Micheals \\ Kayee Kwong \\ Gregory P. Fiumara \\ Information Access Division \\ Information Technology Laboratory
}

This publication is available free of charge from:

http://dx.doi.org/10.6028/NIST.IR.8003

May 2014

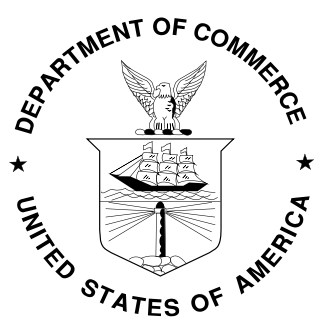

U.S. Department of Commerce

Penny Pritzker, Secretary

National Institute of Standards and Technology

Patrick D. Gallagher, Under Secretary of Commerce for Standards and Technology and Director 


\section{DISCLAIMER}

Certain commercial entities, equipment, or materials may be identified in this document in order to describe an experimental procedure or concept adequately. Such identification is not intended to imply recommendation or endorsement by the National Institute of Standards and Technology, nor is it intended to imply that the entities, materials, or equipment are necessarily the best available for the purpose. 


\section{EXECUTIVE SUMMARY}

In this report, we describe in detail the design and usability testing of a touchscreen interface for multimodal biometric capture, an application called WSABI, Web Services for Acquiring Biometric Information. The application code is publicly available online at https://github.com/NIST-BWS/wsabi2. The interface is a tablet-based reference application for the Web Services for Biometric Devices (WS-BD) protocol. Just as WS-BD specifies a method of communication between client and sensors (i.e., machine-to-machine communication), WSABI provides a consistent and modalityindependent method of interaction between human operators and sensors (i.e., human-tomachine communication). The interface's common capture cards look and function the same across all modalities and all sensors; regardless of the biometric modality or sensor, users perform the same actions to capture, annotate, clear, and retake biometric data. Similarly, users perform the same method of sensor setup regardless of the biometric modality or sensor. Most importantly, this design and functionality has been tested and found successful with real users, participants ranging in age, education, and tablet experience, who were unfamiliar with biometrics and given no training on the application. Given the success of the user-centered WSABI design, the National Institute of Standards and Technology (NIST) recommends that requirements for new systems include the guidelines in the "Design Recommendations" section of this document; including requirements will yield a more effective and efficient user interface for biometric operators, saving both time and money on operator training. 


\section{CONTENTS}

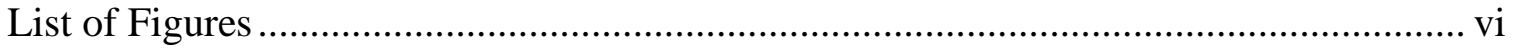

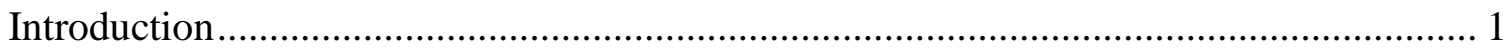

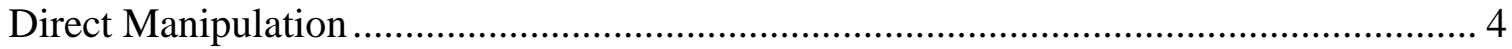

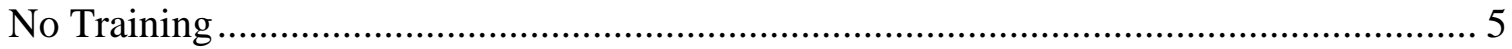

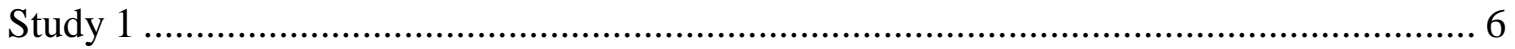

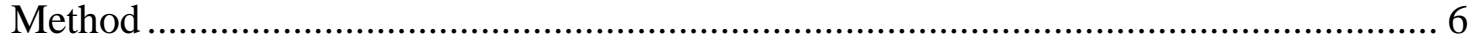

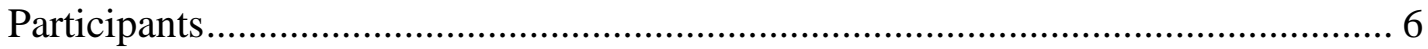

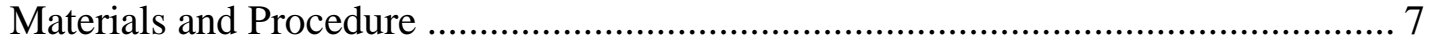

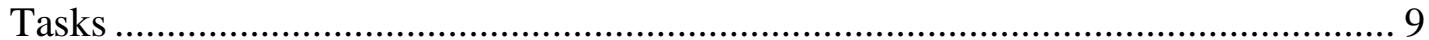

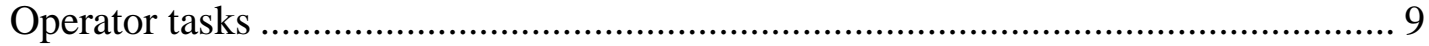

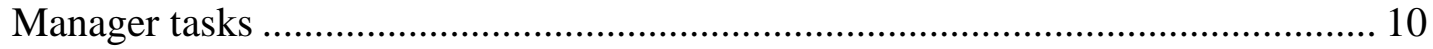

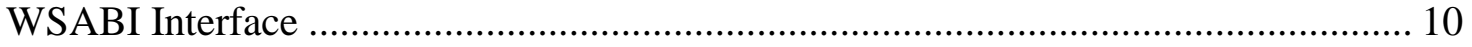

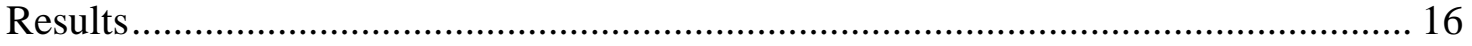

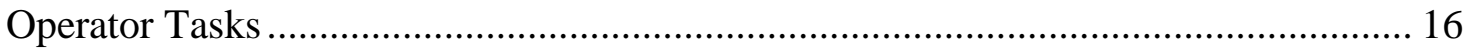

Multi-modal capture

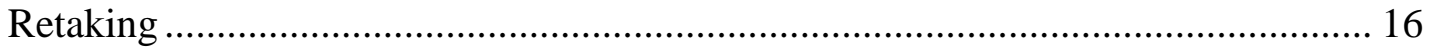

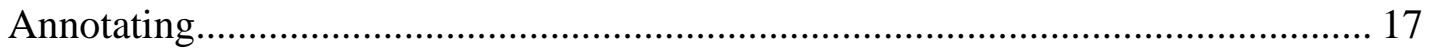

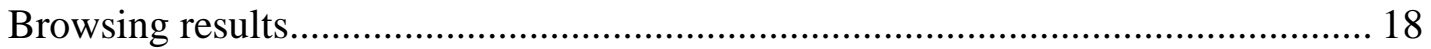

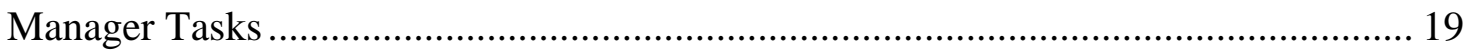

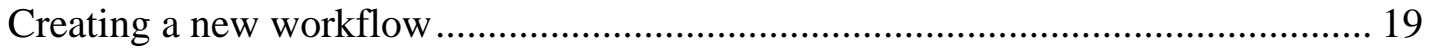

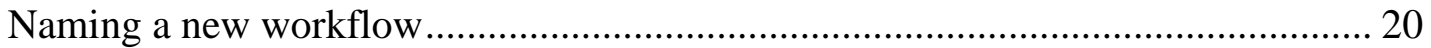

Adding items to a new workflow .................................................................... 20

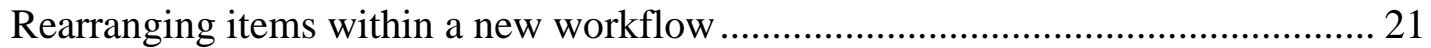

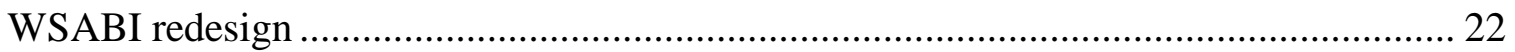

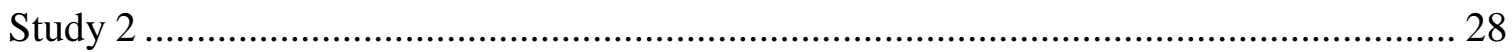

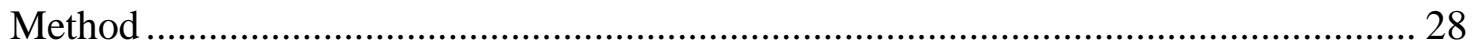

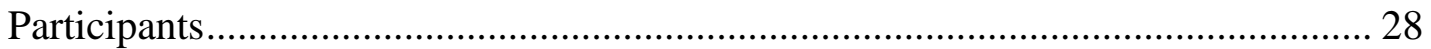

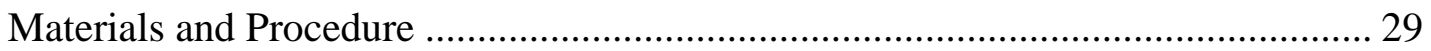

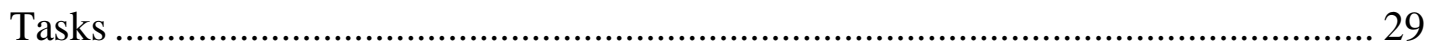

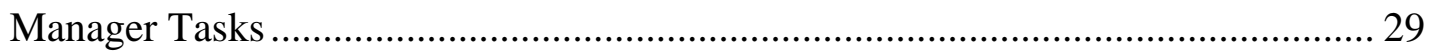

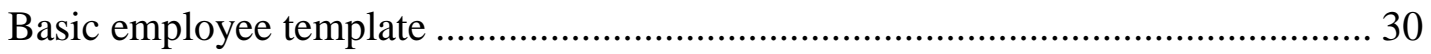

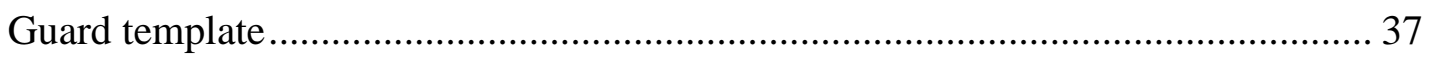

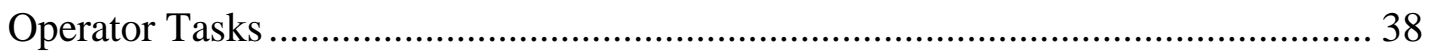




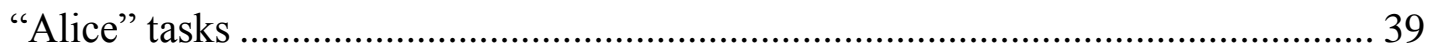

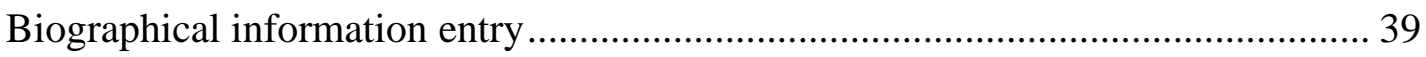

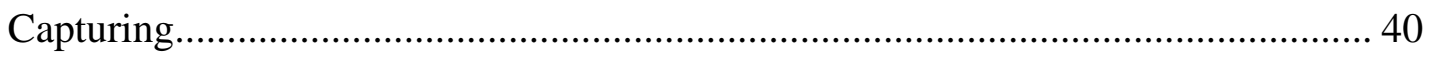

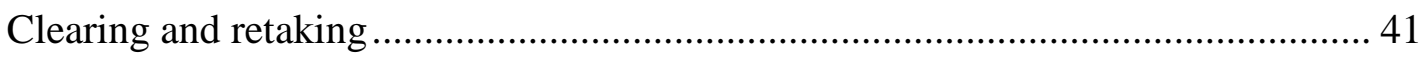

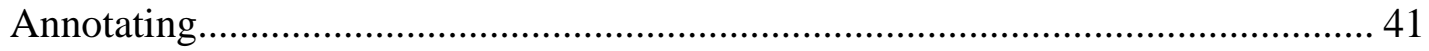

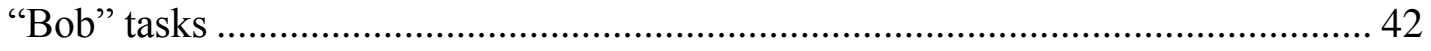

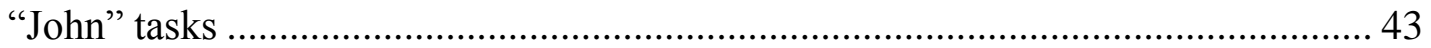

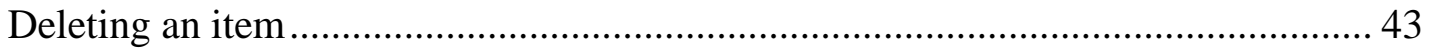

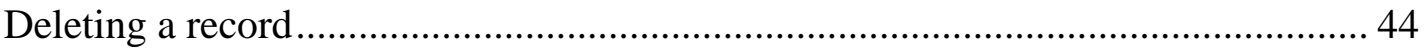

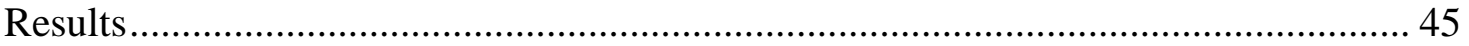

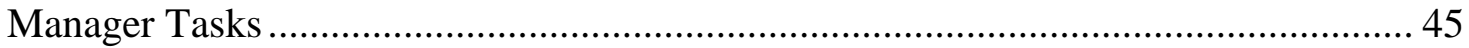

Basic new employee template............................................................................. 45

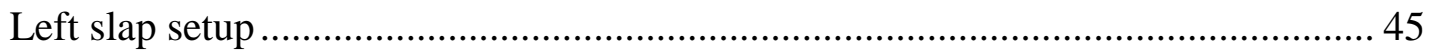

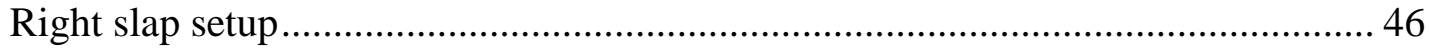

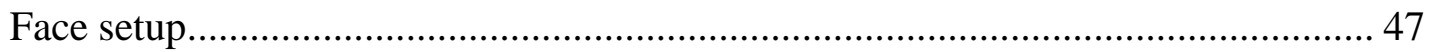

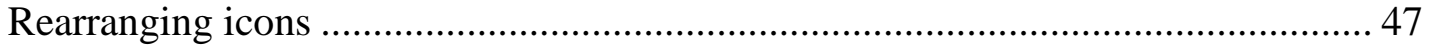

Guard template

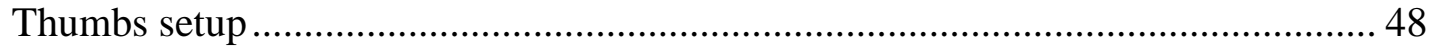

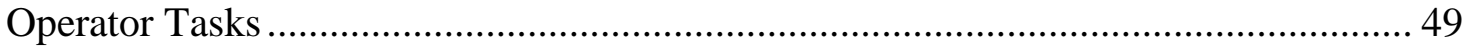

Biographical information entry ………………………...................................... 49

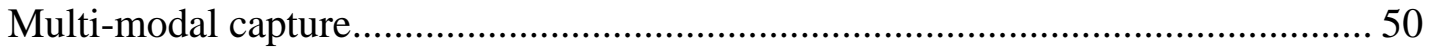

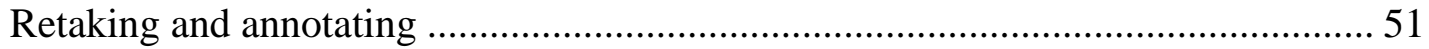

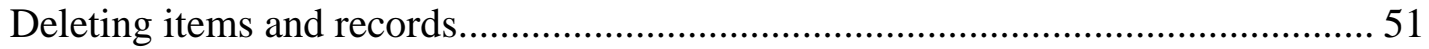

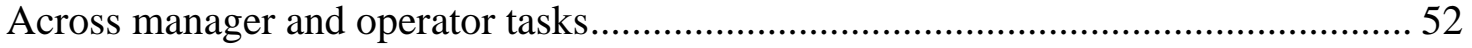

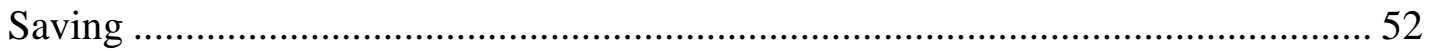

Discussion and Conclusions ................................................................................. 53

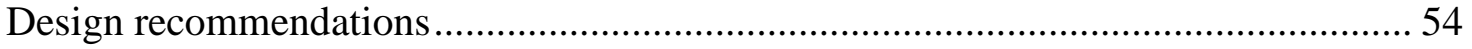

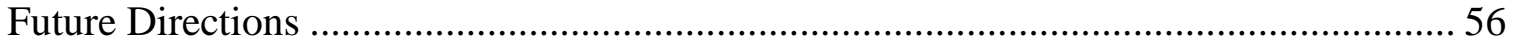

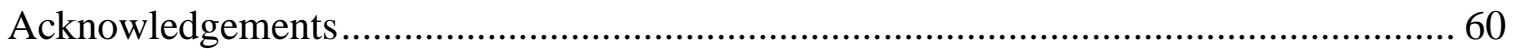

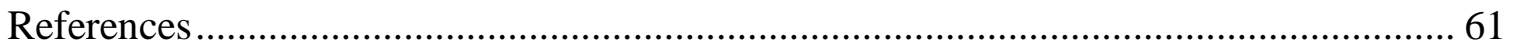

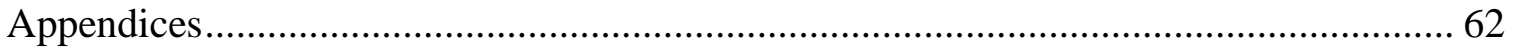




\section{LIST OF FIGURES}

Figure 1. WSABI main screen, common capture card highlighted. ................................ 11

Figure 2. Partial screenshots, common capture card(s). .............................................. 12

Figure 3. Reverse side of a capture card (not to scale)................................................... 13

Figure 4. Operator task, incorrectly completed. ……………......................................... 13

Figure 5. Partial screenshot, operator task, correctly completed. ..................................... 14

Figure 6. Graphical workflow builder. ………………........................................... 14

Figure 7. Sensor popover in workflow builder........................................................ 15

Figure 8. Manager task, completed......................................................................... 15

Figure 9. Collections in non-editable (left) and editable states (right)............................ 18

Figure 10. Manager task, create new workflow......................................................... 19

Figure 11. Scrollable list within a workflow item. ....................................................... 22

Figure 12. Front and back of common capture card in WSABI redesign......................... 22

Figure 13. Partial screenshot of biographical information section. ............................... 23

Figure 14. Multiple records, named record, and icons in redesigned WSABI. ................ 24

Figure 15. Common capture card popover, connected to compact representation of its

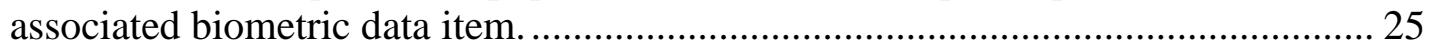

Figure 16. WSABI start screen for manager tasks in Study 2. ...................................... 30

Figure 17. First and second screens of sensor walkthrough: modality (left) and submodality (right) selection. ................................................................................. 31

Figure 18. Third screen of sensor walkthrough: add a new sensor................................. 32

Figure 19. Fourth and final screen of sensor walkthrough: sensor information............... 33

Figure 20. Active person record, after configuring a new sensor to capture a left slap. .. 34

Figure 21. Recent sensors list on third screen of sensor walkthrough............................. 34

Figure 22. Partial fourth screen of sensor walkthrough during right slap setup............... 35

Figure 23. Active person record, after right slap setup.................................................. 36

Figure 24. Face submodality selection on second screen of sensor walkthrough. ........... 36

Figure 25. Active person record, after configuration of face capture............................... 37

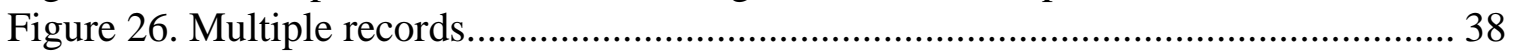

Figure 27. Biographical information task completed. .................................................... 40

Figure 28. "Waiting for sensor" message during capture................................................ 40

Figure 29. Clearing an image................................................................................... 41

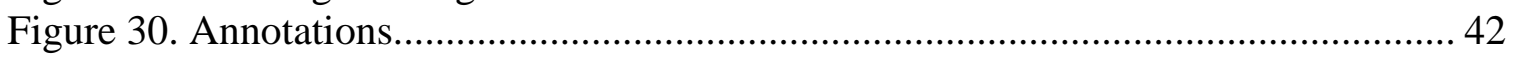

Figure 31. Delete-item task, editable (top) and non-editable (bottom) states................... 44

Figure 32. Delete-record task................................................................................ 44

Figure 33. Edit/Done buttons and modal states for collection-deletion in Study 1 (top) versus item-deletion in Study 2 (bottom)............................................................. 52 


\section{INTRODUCTION}

Currently, biometric devices are typically operated by proprietary software and drivers. Two problems arise from each device having its own vendor-specific software. First, operators require substantial training on each device, with software training for a single device ranging from four to six hours per operator (R. Bowlen, FBI, personal communication, November 30,2012). With hundreds of devices, this adds up to significant money and time spent on operator training for the software alone, not including training on how to take proper prints and troubleshoot devices. Switching vendors means retraining those operators, even though their actual task has not changed. Second, capturing a multi-modal series of biometrics (e.g., fingerprints, iris, face) is a difficult task even for experienced operators, as they must interact with a different piece of software to control each device. These software differences mean that even when operator tasks (e.g., capture data, annotate, clear, retake) are conceptually identical across modalities and sensors, they can be procedurally quite different. The expense of having to train and retrain operators to complete differing procedures for identical tasks might be greatly reduced by making sensors interoperable. While there are obviously costs associated with creating interoperable frameworks, the long-term benefits outweigh the up-front costs.

Until recently, interoperable frameworks for biometric capture were virtually nonexistent. In 2006, the National Science and Technology Council Subcommittee on Biometrics began the National Biometric Challenge to develop middleware techniques and standards to address the interoperability shortcoming. In response, the NIST Biometric Clients Lab developed MBARK, the Multimodal Biometric Application 
Resource Kit (http://mbark.nist.gov). MBARK allowed different devices to talk to the same "middleman," codifying a workflow structure for performing a series of captures. With MBARK, operators no longer had to switch between different pieces of software: the same user interface controlled a variety of sensors from a single desktop PC. While MBARK offered a unifying solution to the problem for Microsoft Windows desktop PCs, technology trends have since shifted away from the desktop environment and toward mobile touchscreen computing. For example, Apple has already sold twice as many iOS devices (i.e., iPads, iPhones, and iPod Touches) as all desktop Macs in history (Dediu, 2012).

To address the widespread shift towards mobile computing, the NIST Biometric Clients Lab began work on a standard protocol, known as Web Services for Biometric Devices (WS-BD), to allow interoperable communication between software running on nearly any platform and nearly any kind of biometric device. Crucially, the protocol allows for both driver-free and wireless communication - both important factors in the world of mobile computing devices. WS-BD makes use of existing web technologies (HTTP, REST-style interaction, and basic XML) to enable this communication. Any device that can "talk the web" can control a WS-BD-enabled device. WS-BD was published as NIST Special Publication 500-288, and work is ongoing to make it an international standard through the International Organization for Standardization (ISO) and the Organization for the Advancement of Structured Information Standards (OASIS). The WS-BD protocol is platform- and operating-system agnostic, providing a common communication that allows physical separation between the client and sensors. The NIST 
Biometric Clients Lab designed an iPad ${ }^{1}$ application, called WSABI (Web Services for Acquiring Biometric Information) to exercise the WS-BD protocol in a reference application. WSABI allows the user to wirelessly control different sensors and capture biometrics from multiple devices. Just as WS-BD specifies a common communication protocol between client and sensor, WSABI gives the user a common interface to those sensors. We designed the interface with two main goals in mind: to leverage the inherent direct manipulation of a touchscreen device, and as much as possible, to require no training with the interface — even for novice users with no prior iPad or biometric experience. To determine whether these design goals were indeed realized, we conducted formative usability testing of the WSABI interface. Based on our first round of usability testing, we significantly redesigned the interface and conducted a smaller second round of testing with the new interface; the original interface and redesign are described in Study 1 and Study 2 Method sections, respectively. The focus of this work was testing the usability of the WSABI reference application for acquiring multi-modal biometrics; the sensors themselves were not tested, nor did we test the biometric data submission process. We focused on usability for the operator during the acquisition process. While previous work (Theofanos, Stanton, and Wolfson, 2008) has focused largely on usability for the end users whose biometrics are being captured, there are additional users who must also be considered, such as those operating the various biometric sensors during the capture process. Our work contributes to the biometrics community specifically by addressing this user group, while also contributing to the larger HCI (human-computer interaction) field by informing general design of mobile touchscreen interfaces. Our work

\footnotetext{
${ }^{1}$ Note that although the WSABI reference application was designed for an iPad, any good tablet would work.
} 
complements existing best practice recommendations for capture, use, security, and transmission of mobile identification data (Orandi and McCabe, 2009) by adding user interface guidelines for touch-and-gesture-based biometric clients; our guidelines are based on formative usability testing results of the WSABI reference application. The application code is publicly available online at https://github.com/NIST-BWS/wsabi2.

\section{DIRECT MANIPULATION}

Touchscreen devices support direct manipulation by removing multiple levels of abstraction from user-device interactions. In a desktop paradigm, users operate a separate physical device (e.g., mouse, trackpad, head-tracker, eye-tracker) to control a representation of their hand on the screen (i.e., the mouse pointer). In contrast, the touchscreen paradigm removes this representational abstraction: users interact directly with the touchscreen via their own hands. A more subtle way in which touchscreens remove abstraction is by allowing users to act directly on an object rather than asking the system to perform the action for them. For example, touchscreens support using pinch/reverse pinch to directly resize an image, rather than selecting an image and using menu options or icons to then resize the image. The touchscreen interaction paradigm makes frequent use of gestures that parallel the physical world, such as picking up and moving an object (drag and drop), and pushing something out of the way or pulling something towards you (natural scrolling). While other touchscreen gestures do not necessarily parallel the physical world (e.g., swipe to enable delete options or press-andhold to activate a contextual menu), they are motorically simple to execute and easy to remember once learned. Gestures that parallel the physical world are implemented similarly across a wide variety of touchscreen devices, whereas actions without such realworld parallelism are more likely to be platform- or device-specific. Well-designed touchscreen user interfaces take advantage of the years of natural control and experience 
people have using their hands to physically interact with —-i.e., directly manipulate — the world around them. This makes them more intuitive for all users, and less intimidating for novice users especially, regardless of age or previous computer expertise.

\section{NO TRAINING}

The very nature of direct manipulation can drastically reduce the amount of user training required. This is especially true for touchscreen-native operating systems (e.g., iOS and Android), as opposed to those systems that simply shrink a previous desktop application to fit upon the reduced screen real estate of a smaller touchscreen device. Using a touchscreen-optimized operating system, iOS, is one way in which WSABI supported reduced user training. By leveraging the iPad's inherent direct manipulation style, little - if any - training on basic interaction gestures is needed. However, simply moving to a touchscreen user interface is insufficient to guarantee zero user training will be necessary. The application and interface must be appropriately designed to truly leverage direct manipulation's intuitiveness and consistency. To this end, we designed WSABI as a common capture interface that would be accessible to a novice. Multimodal capture is difficult to implement technically, but from a user's perspective, all biometric captures should involve fundamentally the same actions. WSABI's design revolved around this idea that users should not have to learn a new user interface per sensor or modality. By designing a capture interface that was consistent across sensors and modalities (see figures in Method section below), we hoped to further reduce user training requirements. 


\section{STUDY 1}

Method

\section{Participants}

Participants consisted of 16 NIST employees, primarily administrative personnel recruited via email. Participants volunteered their time for the half-hour usability study. The only eligibility requirement was a limited prior knowledge of biometrics. Note that it was not possible to recruit participants with zero biometric experience, as Personal Identification Verification (PIV) badge enrollment requires NIST employees to give a full set of prints. iPad familiarity was not required.

Of the 16 total participants, four were pilot participants (two male, two female), whose data are not included in the following results due to extensive prior experience with biometrics and/or user interface design and testing. All 12 remaining participants were female. Participants were fairly diverse in terms of age, education, and prior touchscreen experience. Age ranged from (30 to 39) to (60 to 79) years; education ranged from high school to Bachelor of Arts/Bachelor of Science (BA/BS) and Master of Arts/Master of Science (MA/MS); and touchscreen experience ranged from novice to expert. Using a Likert scale ranging from 1 to 5 , with 1 being "not at all familiar" and 5 being "very familiar", participants were asked to rate their familiarity with using touchscreens to interact with a computer or phone.

Table 1. Participant ages.

\begin{tabular}{|c|c|}
\hline Number of participants & Age range (years) \\
\hline 1 & $30-39$ \\
\hline 6 & $40-49$ \\
\hline 3 & $50-59$ \\
\hline 1 & $60-79$ \\
\hline 1 & no response \\
\hline
\end{tabular}


Table 2. Participant education.

\begin{tabular}{|c|c|}
\hline Number of participants & Education \\
\hline 4 & high school \\
\hline 4 & BA/BS \\
\hline 3 & MA/MS \\
\hline 1 & no response \\
\hline
\end{tabular}

Table 3. Participant touchscreen familiarity ( $1=$ not at all familiar, $5=$ very familiar).

\begin{tabular}{|c|c|}
\hline Number of participants & Familiarity \\
\hline 1 & 2 \\
\hline 5 & 3 \\
\hline 5 & 4 \\
\hline 1 & no response \\
\hline
\end{tabular}

\section{Materials and Procedure}

The study was conducted in the Biometric Clients Lab (BCL) at the NIST

Gaithersburg campus. A second generation Apple iPad running iOS 5 was connected via Wi-fi to multiple sensors that "spoke" WS-BD (Crossmatch Guardian fingerprint scanner, Logitech webcam), which were connected via Universal Serial Bus (USB) to a desktop PC running the WS-BD service on Windows XP. The WS-BD service was written by NIST BCL employees. The WS-BD specification enabled a standard communication between the collection device (iPad 2 running the WSABI application) and the sensors, and allowed the sensors to be physically separate from the device. A Panasonic HDC-TM900, 1080p video camera was mounted on a tripod and used to capture video recordings of the testing sessions. The tripod and camera were positioned behind and to the left of participants such that the camera focus was on the tablet and 
sensors; participant faces were not recorded. Backup audio recordings were captured using the iTalk application by Griffin, installed on an iPhone 4, located on a table (180 $\mathrm{cm} \times 60 \mathrm{~cm}$ ) in front of participants. Audio and video recordings were supplemented by researcher notes, taken both during and after each study session. There was one active researcher and one observer. The former interacted directly with participants, while the latter sat at the back of the room (out of participant sight) to observe and take additional notes. The observer did not interact with participants, although they were informed of his presence. The researcher was seated to the right of participants; a line of black electrical tape divided the shared table into roughly equal halves. The sensors were located on the right side of the table, in front of the researcher, while the iPad was located on the left side of the table, in front of participants. The sensor platen and iPad were both cleaned before the arrival of each new participant.

Upon arrival, participants were greeted and shown to the table, where they sat for the remainder of the study session. After completing the informed consent procedure, they were given a short paper-based demographic questionnaire (Appendices 1 and 2, respectively). The researcher then gave a verbal overview of the study. Participants who had not previously used an iPad or similar device were shown a short general iPad video collage created specifically for this study. (Since the video contained only "novice" gestures, it was not shown to experienced participants.) The one minute video comprised Apple video clips (available from www.apple.com) showing basic gestures like tapping, flicking, scrolling, and zooming. This was done to provide all participants - even those with zero iPad experience - some level of familiarity with basic touchscreen interaction. This allowed us to better differentiate between usability of the WSABI application versus that of the iPad itself; while in some cases the two may be inextricably intertwined, we wanted to disentangle the effects as much as possible.

After the study overview and iPad video, the researcher defined the terms biometric, sensor, and workflow (see Study 1 Scripts, Appendices 4 and 5), then showed 
participants the two sensors (fingerprint scanner and webcam). The researcher demonstrated different types of fingerprints - flat, rolled, both thumbs, single finger, slap - on the fingerprint scanner. Particular emphasis was given to the slap (all four fingerprints from a given hand, taken simultaneously), as it was used repeatedly in the testing tasks. Just prior to beginning the tasks, the researcher turned the iPad on, launched the WSABI application, and handed participants the device. The iPad's orientation lock was on and all participants used the iPad in portrait mode.

\section{Tasks}

All participants used the same iPad to complete two sets of tasks, "operator" and "manager" tasks. Task order was counterbalanced: half of participants completed the operator tasks first, while the other half completed the manager tasks first. The researcher followed a script (Appendices 4 and 5) for each task set. Aside from explicitly defined terms (biometric, sensor, slap, and workflow), the testing script was written to avoid domain-specific technical terms where possible. However, note that for ease of discussion, such terms appear here.

\section{Operator tasks}

For the operator tasks, participants played the role of an operator responsible for taking employees' biometrics. The researcher played the role of presenter, acting as two employees, Alice and Bob. ("Presentation" is defined as the display of biometric characteristics to a sensor. Micheals, Stanton, Theofanos, \& Orandi, 2006.) Participants were asked to use the CrossMatch scanner to collect Alice's left slap print, then retake her left slap. Next, they were asked to use the webcam to collect Alice's face image, followed by the CrossMatch to collect her right slap print. They were interrupted during the collection of the right slap, and asked to retake Alice's face image. Finally, they were asked to mark Alice's left slap print to indicate the index fingerprint could not be 
acquired (Alice was wearing a bandage). Participants then captured Bob's left slap and face image, without being asked to retake anything. However, during the capture of Bob's right slap, participants were told Bob was not feeling well and could not continue, then asked what they would do in this situation. After Bob's biometrics had been recorded, participants were asked to look at the results they had taken in more detail. In this final part of the operator task set, they were asked to open a left slap image in WSABI's full-screen image viewer, zoom in to see it larger, browse to the next image, return to the previous image, then return to the main interface.

\section{Manager tasks}

For the manager tasks, participants played the role of a manager, setting up the system so others could collect data. They were asked to make a new workflow, then call the workflow "Test Number [their participant number]". They were asked to set up the workflow so that it used, in this order: 1) the CrossMatch sensor to capture a left slap, 2) the CrossMatch sensor to capture a right slap, and 3) the webcam sensor to capture a face image. Next, participants were asked to rearrange the steps inside the workflow so that the face image would be captured before the right slap.

After participants completed both sets of tasks, they were asked four exit survey questions (Appendix 3). Time permitting, the researcher also discussed any additional interface characteristics that seemed problematic during the study session.

\section{WSABI Interface}

The main WSABI screen was divided horizontally into two distinct areas, the top area for capturing and annotating biometrics, and the bottom area for creating and editing new biometric collections (Fig. 1). 


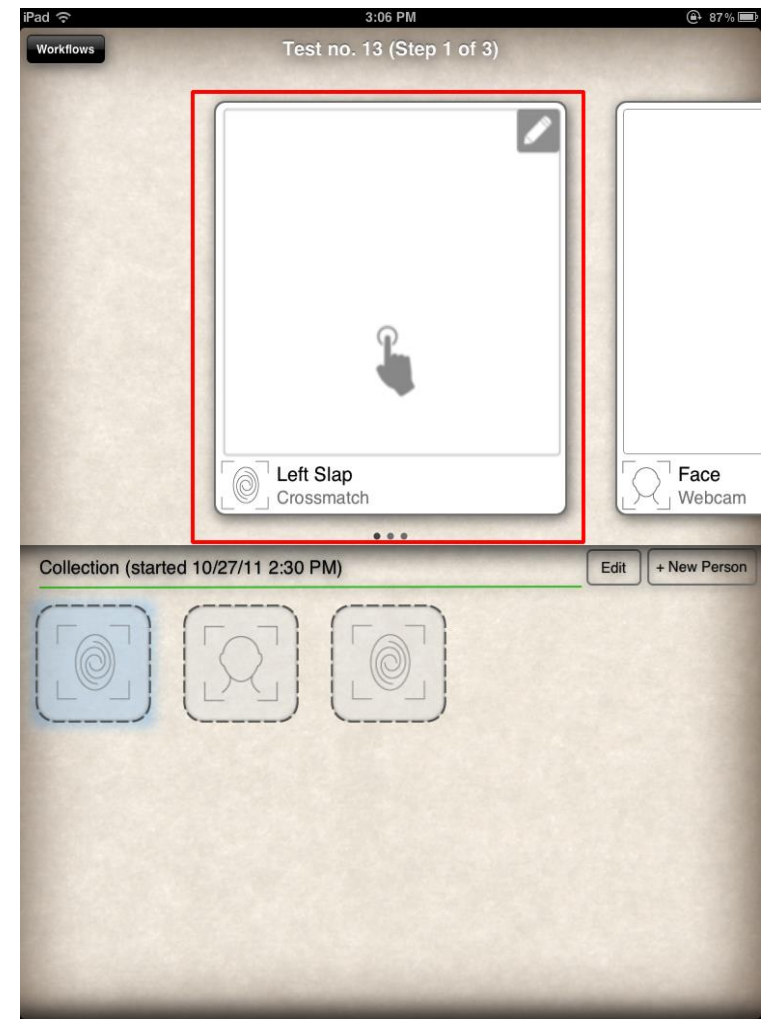

Figure 1. WSABI main screen, common capture card highlighted.

Operator tasks were designed primarily to test the effectiveness of WSABI's "common capture card" (Fig.1 and Fig. 2). Capture cards used action icons (an animated hand to indicate "touch here to start capture" and a [static] pencil to indicate "edit") that were consistent across modalities and sensors. Only the area immediately below the capture area could differ between cards (e.g., "Left Slap, Crossmatch” versus "Face, webcam"). 


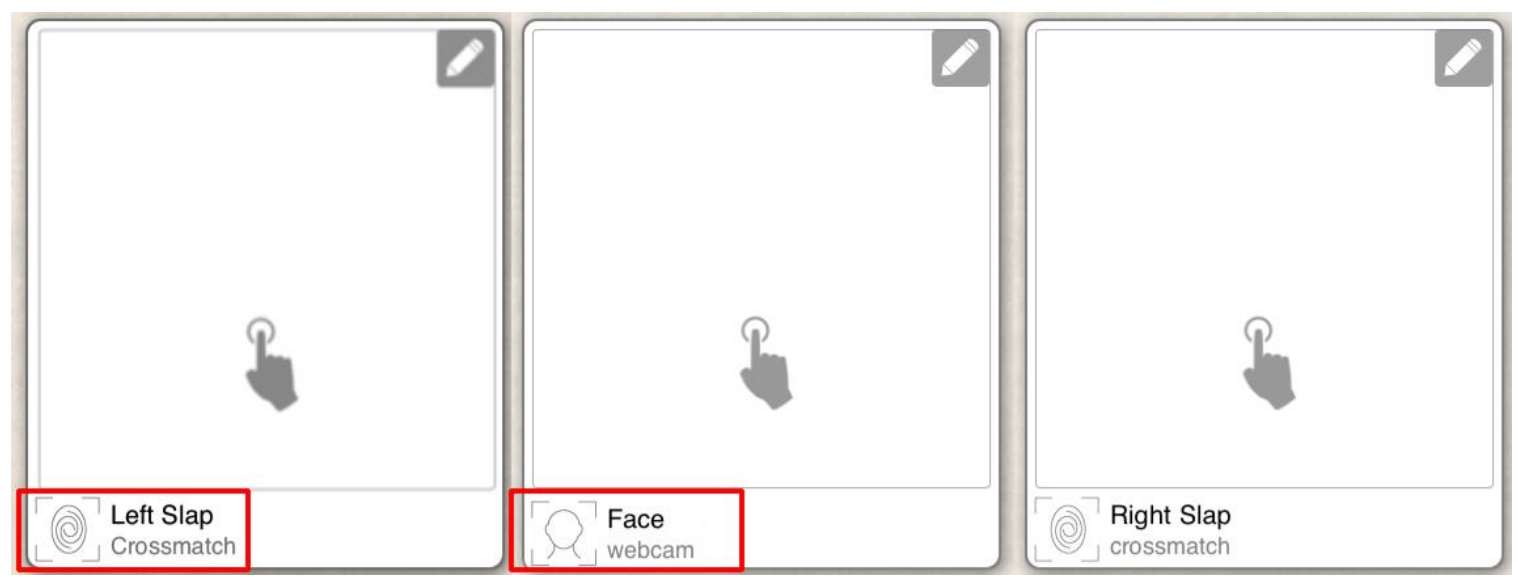

Figure 2. Partial screenshots, common capture card(s).

Just as the WS-BD standard specifies a common communication protocol between client device and sensors, WSABI's capture cards were designed to support consistent interactions between the user and device: users execute the same actions regardless of sensor or modality. Always tap where indicated by the animated hand to start a capture; always tap the pencil icon to edit. More specifically, tapping on the pencil "edit" button in the upper right corner flips the card over (Fig. 3) to allow users to clear results or annotate individual prints or face images. This "flip" animation is common across a variety of iOS applications (e.g., to show track information within the Music app), and illustrates two important design concepts: 1) leveraging the use of animation styles and design elements consistent with other iOS applications, and 2) supporting direct manipulation of objects, in this case by mimicking people's real-world experience with turning over a physical artifact, such as a photo to annotate the date taken on the back. 
This publication is available free of charge from $\mathrm{http}: / / \mathrm{dx}$.doi.org/10.6028/NIST.IR.8003

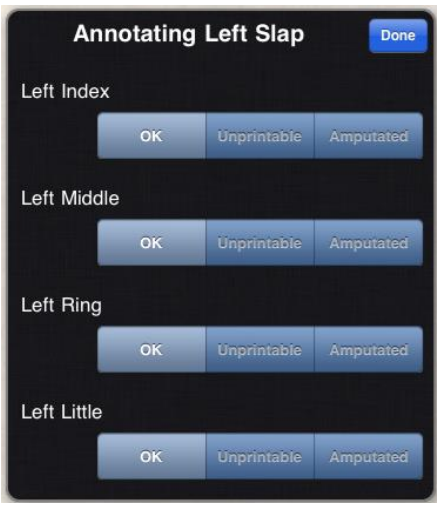

Figure 3. Reverse side of a capture card (not to scale).

After biometrics were recorded, the operator task screen would have two collections, one row each for Alice and Bob (Figs. 4 and 5).

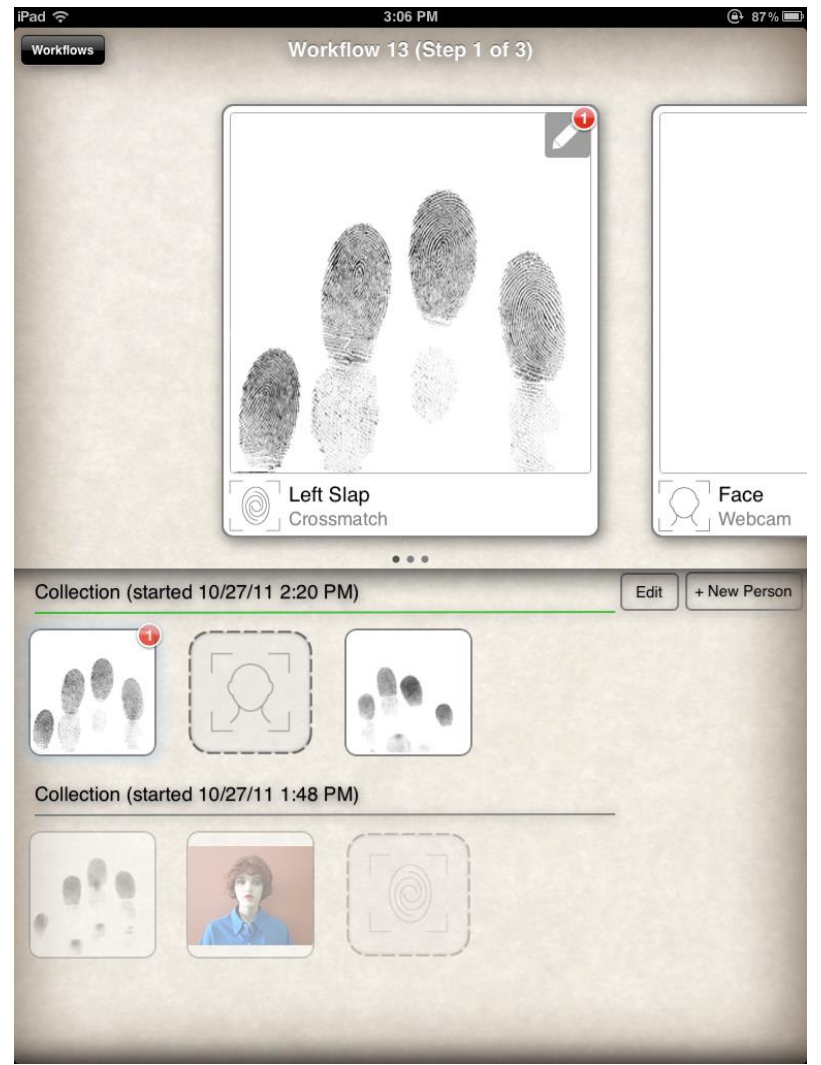

Figure 4. Operator task, incorrectly completed. 


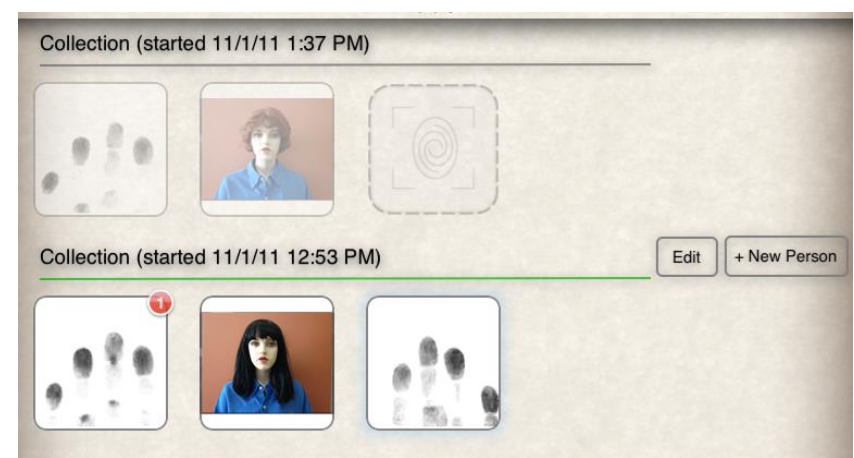

Figure 5. Partial screenshot, operator task, correctly completed.

The manager task set was designed to test the WSABI graphical workflow builder (Fig. 6). Although the concept of a workflow was present in the Multimodal Biometric Application Resource Kit (MBARK), building workflows there was a complicated textual task that required editing raw .xml files; this was accomplished using the Biometrics Client Configuration Language, or BiCCL, a domain-specific language for formally describing biometric client configurations and desired workflows (Aronoff and Micheals, NIST IR 7531). Aside from the change from text-based to GUI-based workflow building, workflows in WSABI are conceptually similar to those in MBARK, i.e., both implement the concept of a "workflow as container."

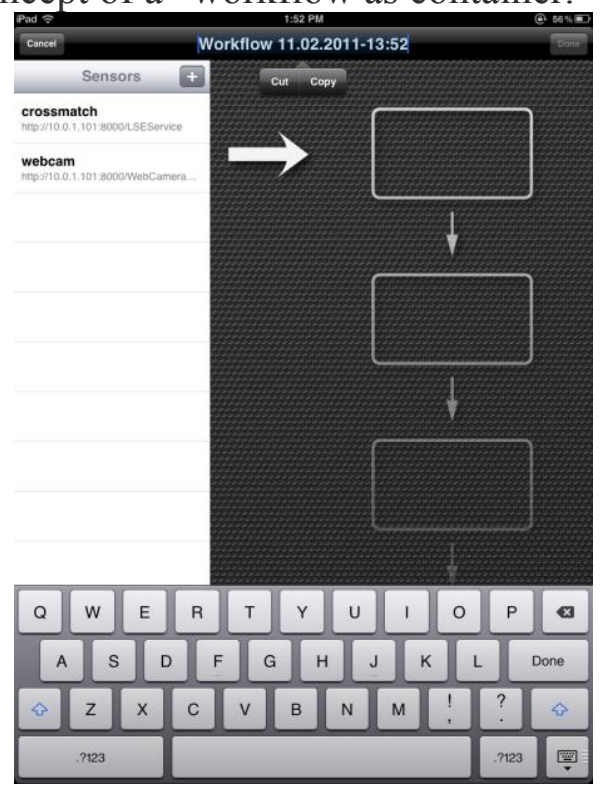

Figure 6. Graphical workflow builder. 
Users could add items to a workflow in two different ways: 1) press and hold to drag a sensor from the shared sensor list on the left to the workflow area on the right, or 2) tap a sensor in the shared sensor list and select "Add to workflow" from the resulting popover (Fig. 7).

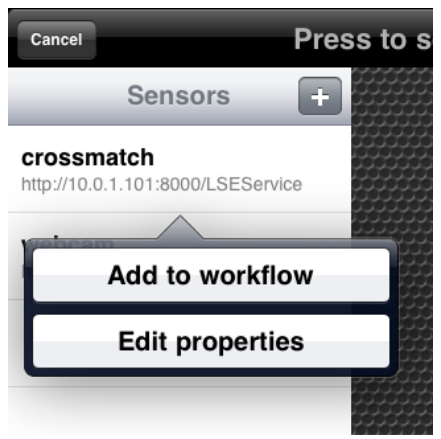

Figure 7. Sensor popover in workflow builder.

Once the manager task set was completed, the empty boxes in the workflow builder area (previous Fig. 6) would then appear as shown in Figure 8.

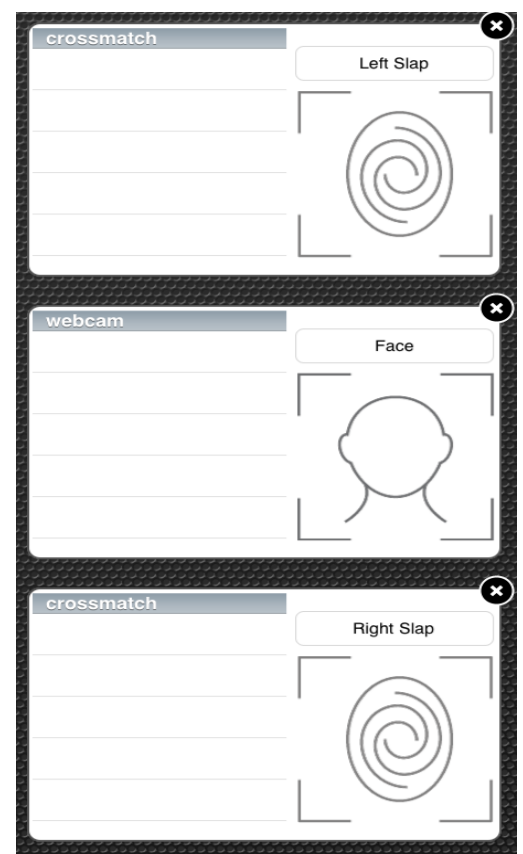

Figure 8. Manager task, completed. 


\section{Results}

Operator Tasks

\section{Multi-modal capture}

Perhaps the single most important result was the success of WSABI common capture cards. Using these capture primitives, all participants were able to record multimodal biometrics from different sensors; no modality was easier or more difficult than another. In some cases, participants instructed the researcher (in the role of Alice) to place her hand on the sensor before starting the capture. When no image appeared on the iPad, participants then realized an action was necessary on their part; they then tapped where indicated by the animated hand on the iPad to start capturing. Some participants initially tapped the small fingerprint icon (in the lower left corner of the capture card) before tapping on the animated hand. Nonetheless, in all cases users successfully recorded biometrics.

\section{Retaking}

Initially, retaking prints and images was accomplished simply by tapping the animated hand a second time. After testing with only a single participant, the destructive nature of this implementation was clearly evident. When a user tapped the icon, it started a new capture immediately, which instantly overwrote existing data. Since this issue was both severe and easily addressed, it was fixed prior to testing with the remaining participants. The issue was addressed by implementing an explicit clearing action before retaking was allowed. Once an image was acquired, the animated hand icon disappeared. To retake an image, the user had to tap the pencil edit button in the top right of the capture card, and select the "clear result" option from the resulting popover. After clearing the existing result, the hand icon would reappear to allow a new capture. The new implementation worked well, preventing users from accidentally overwriting data, while still allowing them to easily clear and recapture data. 


\section{Annotating}

Using a button containing a pencil icon to initiate annotation was successful overall, but there was some confusion over the wording of the annotation choices. For example, many users hesitated to select "unprintable" to indicate that Alice's left index fingerprint could not be acquired (recall that "Alice was wearing a bandaid"). Even in cases where participants expressed this uncertainty, they still correctly selected "unprintable" rather than "amputated." More interesting was the unintended annotation task for Bob's right slap. Partway through Bob's right slap collection, participants were told that he was sick and could not continue. This was intended to prompt use of the "cancel" action. Overwhelmingly, participants attempted to annotate this capture card, explicitly expecting a "notes" field or some other method to indicate why the print was not taken. In several cases, participants marked all four of Bob's fingers as unprintable as a work-around.

The annotation task also illustrated conceptual issues with the Edit and Done buttons on the collection row. Edit and Done are modal: the same button holds both functions. As soon as the Edit button is tapped, items are put in an editable state and the button label changes to "Done". As soon as the Done button is tapped, items are no longer editable and the button label reverts back to "Edit". The modal Edit/Done or Edit/Cancel concept is consistent with the iOS Human Interface Guidelines (HIG) (Apple, 2014). In some cases, participants would tap the Edit button next to the active collection (on the bottom half of the screen), then complete the annotation task on the capture card (on the top half of the screen). Tapping the collection's Edit button was intended to allow deletion of that entire collection, and it remained in this "editable" state (with the standard iOS red deletion circles present, Fig. 9) until the Done button was tapped. 

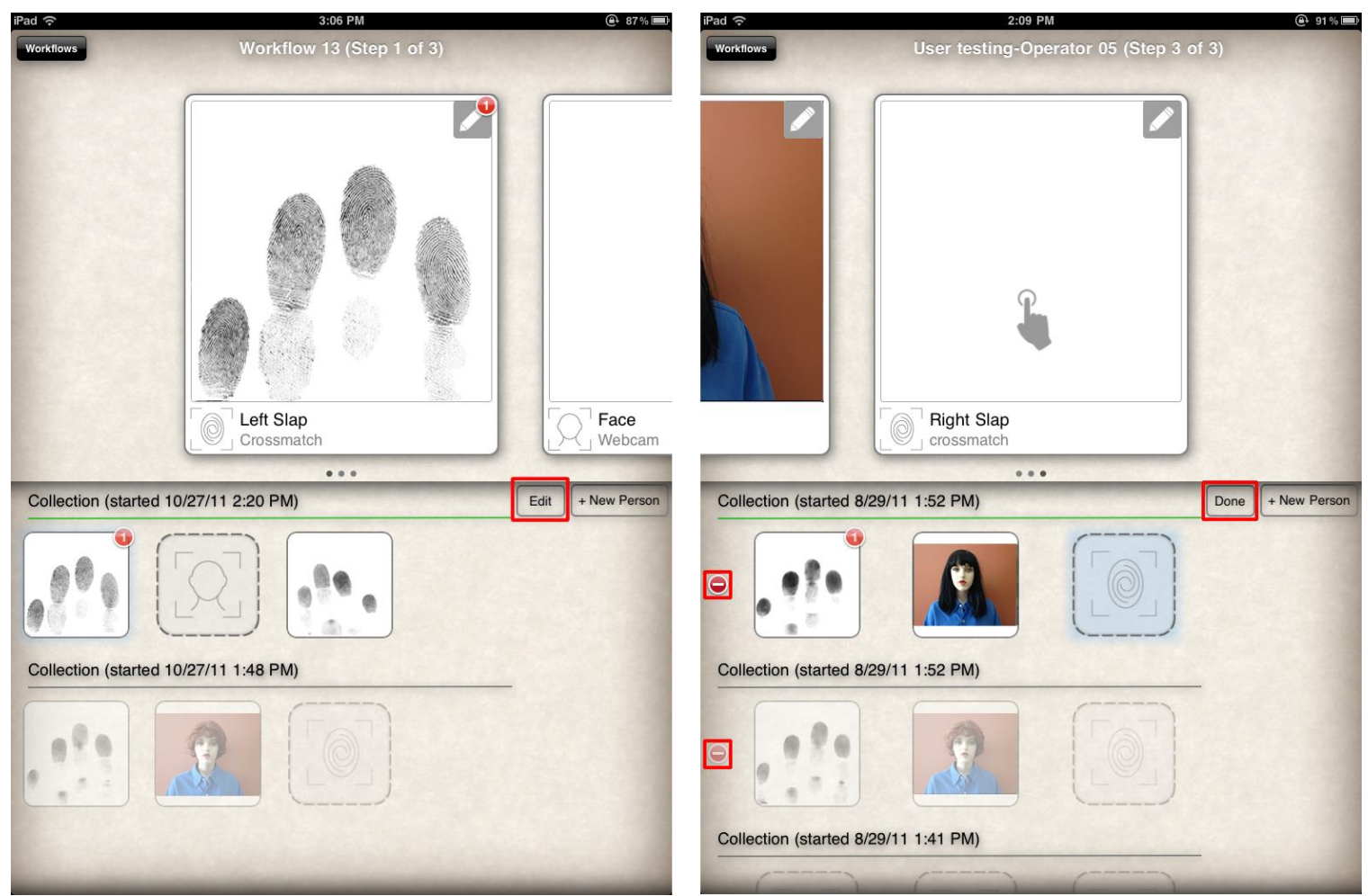

Figure 9. Collections in non-editable (left) and editable states (right).

Some participants tapped the Done button immediately after annotation, whereas others did not tap Done until they were completely "done" with Alice and Bob's capture tasks. The more general desire to indicate "done-ness" by explicitly saving data was common. This is not surprising given that the need to save is deeply ingrained in the traditional desktop paradigm. The saving issue was not unique to the annotation tasks, and also arose during workflow building.

\section{Browsing results}

When asked to open a left slap image full-screen, some participants double-tapped the image and others reverse-pinched. Since WSABI supported multiple interaction gestures, in both cases users successfully opened the image in full-screen mode, regardless of whether they double-tapped or reverse-pinched. Note that if a given result was zoomed in beyond the bounds of the screen, the user could not browse to the next 
image without first zooming out or scrolling to the edge of the zoomed-in image. Several users tried to move to the next image without initially realizing they had to zoom out from the current image view. Some users swiped to browse between captured images, whereas others tapped on the white arrows at the bottom of the screen. Again, since multiple methods were supported, users were successful either way. In contrast, returning to the main interface posed a difficulty for many users. After several seconds of inactivity, the done button and navigation arrows "fade out". Not realizing that they could tap to make the controls reappear, many users pressed the physical iPad home button. While this is an interesting result, it does not seem to be specific to the WSABI interface. The iOS Photos application operates in a similar way, and in fact, the "fade away" and "tap to make reappear" actions were shown briefly in the iPad video.

\section{Manager Tasks}

\section{Creating a new workflow}

In order to access the workflow builder screen, participants had to tap the "Workflows" button on the main operator screen. From the resulting popover, they then had to tap the plus button (Fig. 10).

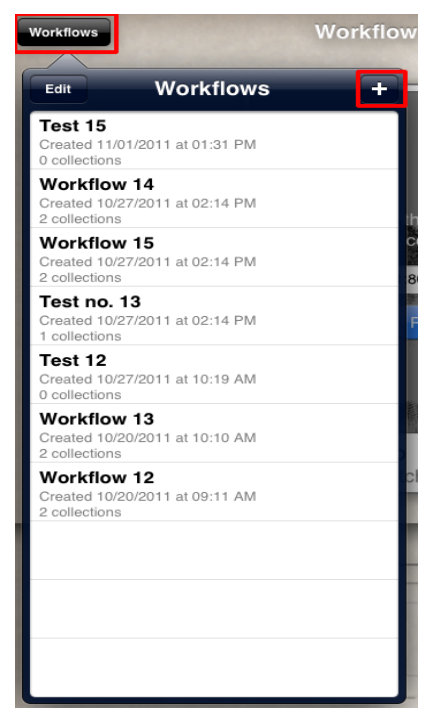

Figure 10. Manager task, create new workflow. 
The workflow button itself was often difficult for participants to find, and once found, the embedded plus button posed even greater difficulty. Within the workflow popover, participants often tapped on the edit button and/or one of the other menu items, which would launch a previous workflow rather than creating a new one. Simply getting to the workflow builder often posed a significant issue and required additional researcher prompting.

\section{Naming a new workflow}

Initially, one could set the title of a new workflow by tapping the title bar within the graphical workflow builder, which would bring up the onscreen virtual keyboard. The first few participants did not notice the "Press to set workflow name" prompt on the title bar, and created a nameless workflow. This was partially addressed by automatically highlighting the title text and bringing the keyboard up as soon as the workflow builder screen was accessed. However, some users still experienced difficulty due to overlooking the virtual keyboard at the bottom of the screen.

\section{Adding items to a new workflow}

As previously described, there were two different ways that items could be added to a new workflow. Each method posed unique difficulties for participants. The first method, drag and drop, suffered from timing implementation issues. The "press and hold" time required to make a sensor "draggable" was too long. Several participants attempted this method using the correct interaction technique, but tried moving the sensor too quickly, i.e., before it was in a "draggable" state. When participants tried this method multiple times without success, the researcher explained the timing lag. If the drag and drop method failed participants yet again, the researcher explained that there was an alternative method, and suggested they try tapping on the sensor. 
The second method, tap and select, gave rise to the most severe errors. As shown in previous Fig. 7, tapping a sensor displayed a popover with two options, "Add to workflow" and "Edit properties." Many participants chose "Edit properties" rather than "Add to workflow," which was the target option. Of those participants, upon reaching the sensor properties menu, a few hit "cancel" and backed out immediately. Others proceeded to unintentionally edit shared sensor properties or even delete a sensor entirely, rather than adding the shared sensor to the workflow they were building. Editing properties belonging to a shared sensor, or deleting a shared sensor, had severe and lasting consequences for any existing workflows using that same sensor. As workflows contain permanent shared representations of sensors, deletion or modification of shared sensors can irreparably break multiple workflows. Finally, in attempting to add an item to the workflow builder, several participants added an entirely new sensor by tapping the plus on the shared sensor list (previous Fig. 7). Even without entering a valid IP address, they were able to add a new sensor to WSABI's shared sensor list.

\section{Rearranging items within a new workflow}

Rearranging items within a workflow was much less problematic than adding them. Participants clearly understood the rearranging task and attempted the appropriate interaction gestures to accomplish it. However, two interface issues prevented many participants from completing the task on their first try: 1) a long drag and drop timing delay, and 2) accidental activation of a scrollable area within the card. As with the drag and drop to add task, the "press and hold" time required to make an item "draggable" was too long. Several participants attempted to rearrange the items using the correct gesture, but tried moving an item too quickly, i.e., before it was in a "draggable" state. In some cases, the gesture resulted in moving a scrollable list within the item itself (Fig. 11). This scrolling list was intended to support recording additional information in the future, i.e., more information than could be displayed in the current item. 


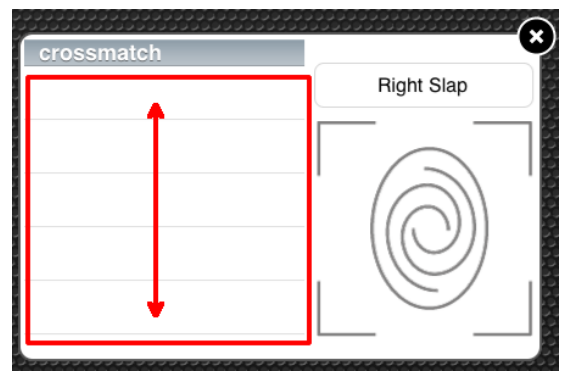

Figure 11. Scrollable list within a workflow item.

\section{WSABI REDESIGN}

There were two primary goals when redesigning the WSABI interface to incorporate Study 1 user testing results: keep what worked well, and fix what didn't. For the operator task set, WSABI's original common capture card and annotation interface worked extremely well. The redesign kept capture and annotation intact as part of a single object, again representing a capture card as an analog of a directly manipulable physical object with a front and a back (Fig. 12).
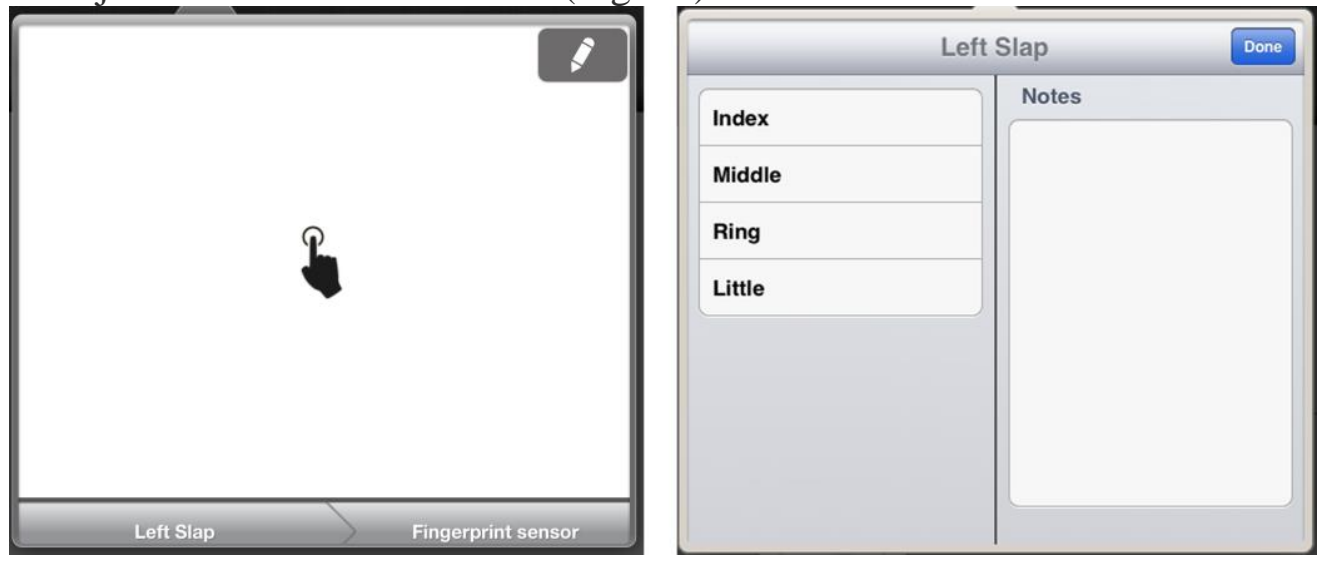

Figure 12. Front and back of common capture card in WSABI redesign.

The redesign implemented a biographical record section (Fig. 13) with specific fields and option choices, such as name, gender, age, hair color, and eye color. The capability to record biographical data - and thereby identify a collection by name or other biographical characteristic - was not previously present. A free-form notes field was also added in the WSABI redesign, in order to address participants' desire to note specifically why a biometric wasn’t captured (i.e., Bob wasn’t feeling well and will return tomorrow). 


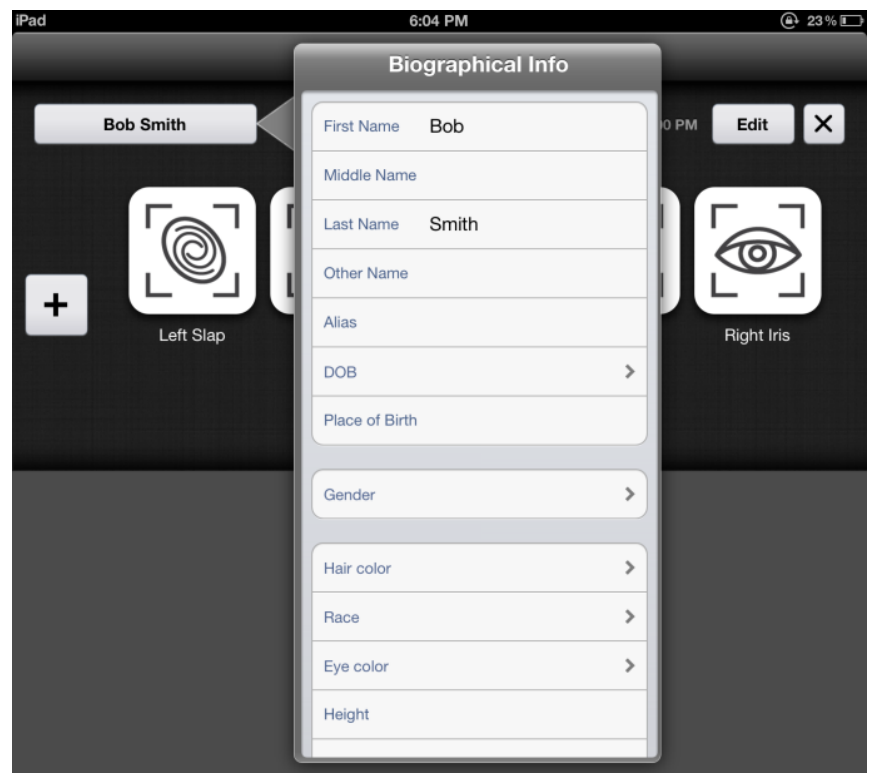

Figure 13. Partial screenshot of biographical information section.

For the manager task set, the redesign sought to address shortcomings found in the workflow creation task. Based on the difficulty and severity of errors found during user testing, the concept of a workflow clearly needed revising. In WSABI's original conceptual model, the workflow was a static "container" for particular sequences of biometric data (e.g., a left slap followed by a right slap followed by a face image). The consequences of a static container model were problematic: any workflow that contained data could not be restructured, because the data already in the container would become invalid. This approach was significantly at odds with the hands-on nature of the touchscreen interface, as well as with user expectations. Consequently, the conceptual approach to capturing multiple biometrics changed significantly in the redesigned interface. In the WSABI redesign, the canonical object became the "person," rather than the "workflow." Each person could then have any sequence of associated biometric data, and each item in that sequence had all the necessary information to communicate with a relevant biometric sensor. 
Simplifying the conceptual model allowed us to map the user interface more closely to that model, as well as to bring direct manipulation philosophies to bear upon the weaknesses of the previous design. In the WSABI redesign, each person was represented by a single row (Fig. 14). All information related to that person was contained in that row. For example, the new biographical information area was available as a selfcontained entry area accessible from a button in the top left; that button's text comprised the name contained in the biographical information area. Each biometric data item appeared as part of a grid within the cell. The compact representations of biometric items from the previous design were reused with one small modification to the finger icon: it was now angled slightly to discourage people from trying to place their thumb there. Recall that while the compact representations largely worked well during user testing, there were several instances where participants erroneously pressed their thumb on the fingerprint icon.

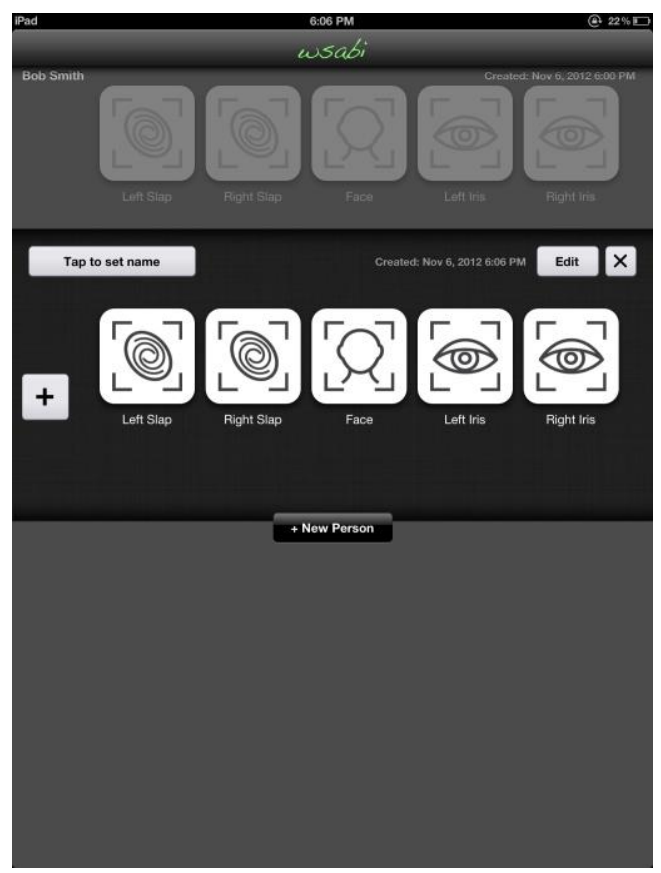

Figure 14. Multiple records, named record, and icons in redesigned WSABI. 
Tapping the compact representation displayed the common capture card within a popover connected to that representation, so that contextual information about the relationship between the capture card and the biometric item was more readily available (Fig. 15). The biometric modality and submodality, as well as the associated sensor, are shown in breadcrumb bars (the arrow-shaped buttons labeled "Right Slap" and "Finger Sensor" in Fig. 15) within the common capture card.

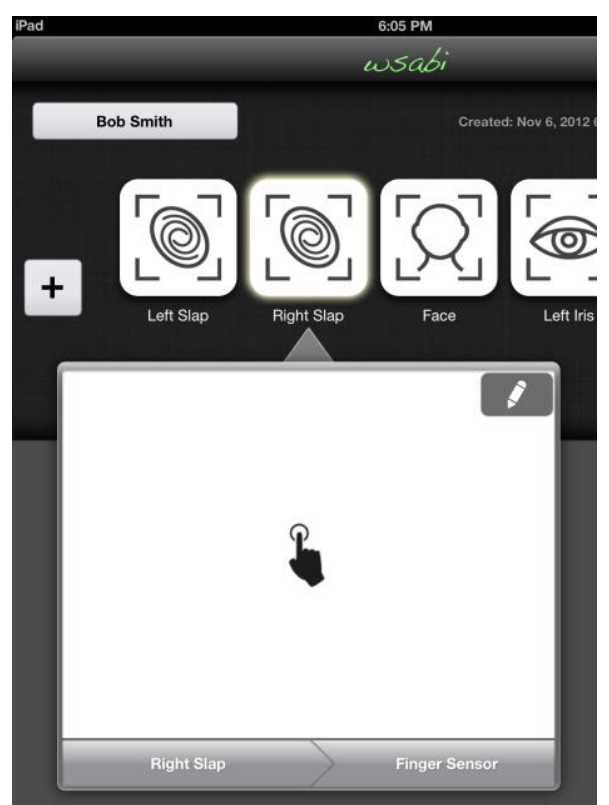

Figure 15. Common capture card popover, connected to compact representation of its associated biometric data item.

With the exception of removing the concept of a static workflow, by far the most significant change in the redesigned WSABI was the new sensor setup walkthrough. The concept of a static workflow made the original interface sensor-centric, requiring users to know names and technical capabilities of a sensor in order to select it from a sensor list (as in previous Fig. 7). If an operator's task is to collect a fingerprint, why show them any sensors that cannot capture fingerprint data? In sharp contrast to the exhaustive list of sensors ever-present in the original interface (i.e., sensor-centric), the redesigned sensor setup process was user- and task-centric, walking users through the process based on 
what biometric information they were trying to collect, and showing only sensors with the capability to collect that type of biometric data. Sensor setup was not tested at all in Study 1; with the original interface, all sensors were set up before testing and participants had to select them from a list on the separate workflow builder screen. In Study 2, the sensor setup process was a primary focus of usability testing; participants had to set up multiple sensors from scratch (described in the Study 2 Task section).

The substantial changes to the user interface and conceptual model meant analogous changes for the underlying iOS code and data model. Whereas the original WSABI data model required a stand-alone, persistent representation of a sensor, the new interface did not. Additional code changes included those necessary to update WSABI to the latest iOS version (at the time, iOS 6.1), as well as the instrumentation of touchlogging. Touch-logging capabilities were added during the WSABI redesign to help capture more fine-grained data, which in turn informed computational cognitive modeling efforts (Greene, Tamborello, and Micheals, 2013; Greene and Tamborello, 2013). To make touch analysis easier and enable potential future automation of analysis, information about the user's interaction with the device was also recorded. A file containing a readable account of the WSABI usage could be offloaded after the participant's session had concluded.

This interaction log was made possible by features inherent to most user interface elements in the iOS software development kit (SDK), specifically by leveraging the iOS responder chain. Many human interactions with a device running iOS create "usergenerated events" in the operating system. Almost any interaction, such as a swipe, touch, or shake, can be considered an event (Apple, 2013). Apple has continued to add new classifications of events with newer versions of their hardware and software. When a user-generated event occurs, the operating system follows a simple path to alert interested parties that the event has taken place, known as traversing the responder chain.

Consider this (extremely simplified) example of touching a button on the screen, 
starting in software. The operating system notifies the currently running application that a touch event occurred at a specific coordinate. That application then traverses the various user interface elements currently displayed on the screen at that coordinate, alerting the backing object that the event has occurred. This starts with the "first responder," which is typically the user interface element most in the foreground of the application. From there, the object may respond to the event, or forward the event to the next interested party.

Under normal circumstances, a developer may very well never have to interact with the responder chains. This is because all iOS SDK user-interface elements handle the standard user-generated events in a predicable way, making possible common-day "event-driven programming." In the case of WSABI, there were certain touch-based interactions we wanted to record in order to enhance the user interface of the application. For the user interface elements and user-generated events we were interested in, a simple line of text was recorded as the responder chain traversed the object. This line would include a description of the user interface element on which the event was triggered, the coordinates of the event in terms of the user interface object and the device, and any other pertinent information depending on the interaction type. Where possible, a gesture recognizer was used for the logging, so as to not have to predict whether or not the usergenerated event should be canceled, dealt with, or forwarded. This critical task was instead left to the SDK element's default value. Gesture recognizers have a property in which they can be simultaneously notified of an event along with their containing object. In the case of the person list, this meant that we could continue to scroll the list while recording the change in position of the list and inferring the user's finger movement, all without causing any undesired visual conflicts. In the end, this allowed a precise readable account of the participant's usage of the application. 


\section{STUDY 2 \\ Method}

\section{Participants}

Unless otherwise noted, Study 2 Method mirrored that of Study 1. Participants consisted of seven NIST employees (six females, one male), five of whom participated in Study 1. Participants were again fairly diverse in terms of age, education, and prior touchscreen experience. Age ranged from 21-29 to 50-59 years; education ranged from high school to $\mathrm{PhD}$; and touchscreen experience ranged from average to expert.

Table 4. Participant ages.

\begin{tabular}{|c|c|}
\hline Number of participants & Age range (years) \\
\hline 2 & $21-29$ \\
\hline 1 & $30-39$ \\
\hline 2 & $40-49$ \\
\hline 2 & $50-59$ \\
\hline
\end{tabular}

Table 5. Participant education.

\begin{tabular}{|c|c|}
\hline Number of participants & Education \\
\hline 2 & high school \\
\hline 3 & $\mathrm{BA} / \mathrm{BS}$ \\
\hline 1 & $\mathrm{MA} / \mathrm{MS}$ \\
\hline 1 & $\mathrm{PhD}$ \\
\hline
\end{tabular}

Table 6. Participant touchscreen familiarity (1=not at all familiar, 5=very familiar).

\begin{tabular}{|c|c|}
\hline Number of participants & Familiarity \\
\hline 3 & 3 \\
\hline 1 & 4 \\
\hline 3 & 5 \\
\hline
\end{tabular}


Note that although Study 2 participants rated themselves as slightly more familiar with touchscreens than did Study 1 participants, of the three who rated themselves as very familiar, only one mentioned being familiar with a tablet.

\section{Materials and Procedure}

An iPad 2 with high-resolution display running iOS 6.1 was used in Study 2. While a Crossmatch fingerprint sensor was used in Study 1, a Suprema fingerprint sensor was used in Study 2. It is important to emphasize that across both studies, we were testing the usability of the WSABI interface and not the sensors themselves. Since the WS-BD protocol is device-agnostic, it was important to use sensors from different manufacturers. In addition to these changes in materials, several modifications were made to the procedure in Study 2. There was no longer an observer in the room during testing; other than the participant, only the researcher was present. The general iPad video comprised of Apple video clips was no longer shown, and the script and background information given were even further reduced in Study 2. For example, the explanation of rolled versus flat fingerprints was not given; since participants were not asked to take rolled fingerprints, this information was superfluous. Manager/operator task roles were no longer counterbalanced in Study 2; since one of the primary goals was now testing the sensor walkthrough, we wanted participants to configure sensors (i.e., the manager tasks) before using them to collect any data (i.e., the operator tasks). In front of both the face sensor and the finger sensor were white placards, each with the respective sensor's network address and name printed in black ink.

\section{Tasks}

\section{Manager Tasks}

Since the concept of a fixed workflow was removed in the redesigned WSABI, rather than using the term "workflow" for manager tasks in Study 2, we used the more 
appropriate term "template." The manager tasks were to set up two templates, one for a "basic employee" enrollment (left slap, right slap, face) and one for a "guard" (same as a basic employee, but with the addition of a thumbs slap). This required configuring two different sensors, a fingerprint sensor and a face sensor (Suprema and Logitech, respectively). While the following sections describe the individual tasks in great detail, it is important to emphasize that participants were not given these step-by-step instructions (see Appendix 6 for Study 2 Script). They were intentionally given as little information as possible. For example, the only instructions they were given for the left slap configuration task were: "The first thing you want to set up is the capability to record a left slap, from this sensor," which was accompanied by the researcher pointing at the Suprema fingerprint sensor.

\section{Basic employee template}

When participants were handed the iPad at the start of testing, the WSABI application only showed a circular plus in the center of the screen (Fig. 16). One must tap this button to start the sensor walkthrough.

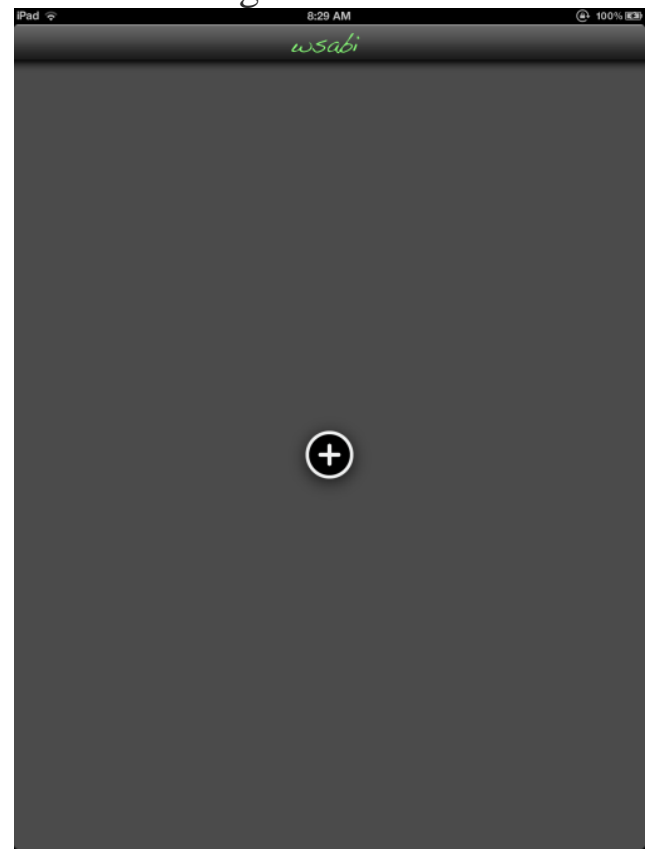

Figure 16. WSABI start screen for manager tasks in Study 2. 
The next required action is a tap on the finger button to select the capture type (biometric modality) on the first screen of the sensor walkthrough, followed by a tap on the left slap button to select submodality on the second screen of the sensor walkthrough (Fig. 17).

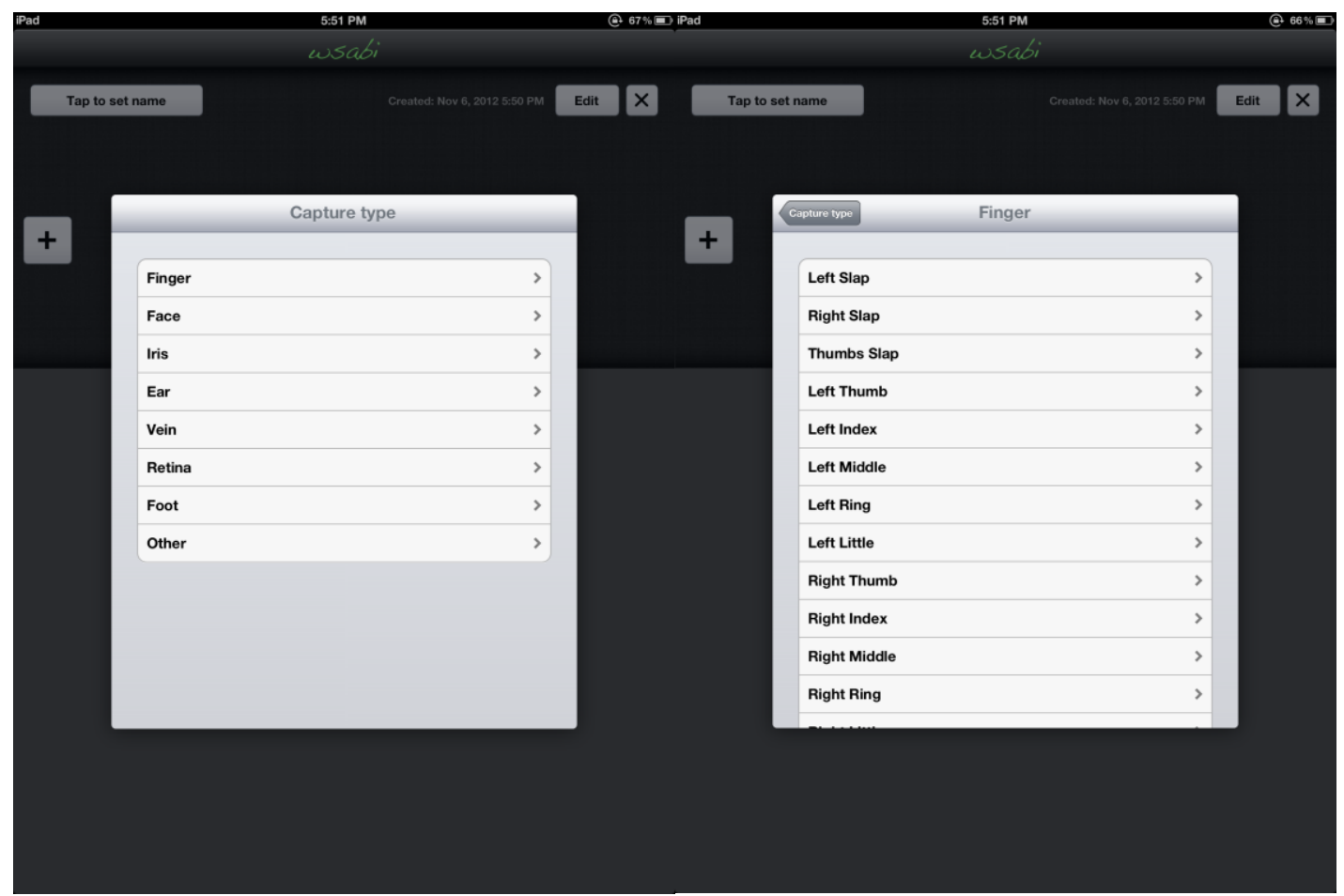

Figure 17. First and second screens of sensor walkthrough: modality (left) and submodality (right) selection.

The previously selected modality appears at the top of the walkthrough, and what functions as the "back" button is instead labeled with "Capture Type" to indicate what tapping it would navigate back to. Again, note that on both the modality and submodality menus, the most frequently used options are at the top of the list. The next required action is a tap on the "add new sensor" button on the third screen of the sensor walkthrough (Fig. 18). Notice the walkthrough is now labeled with the chosen modality and submodality, and the "back" button is again labeled with information on what tapping it navigates back to. 


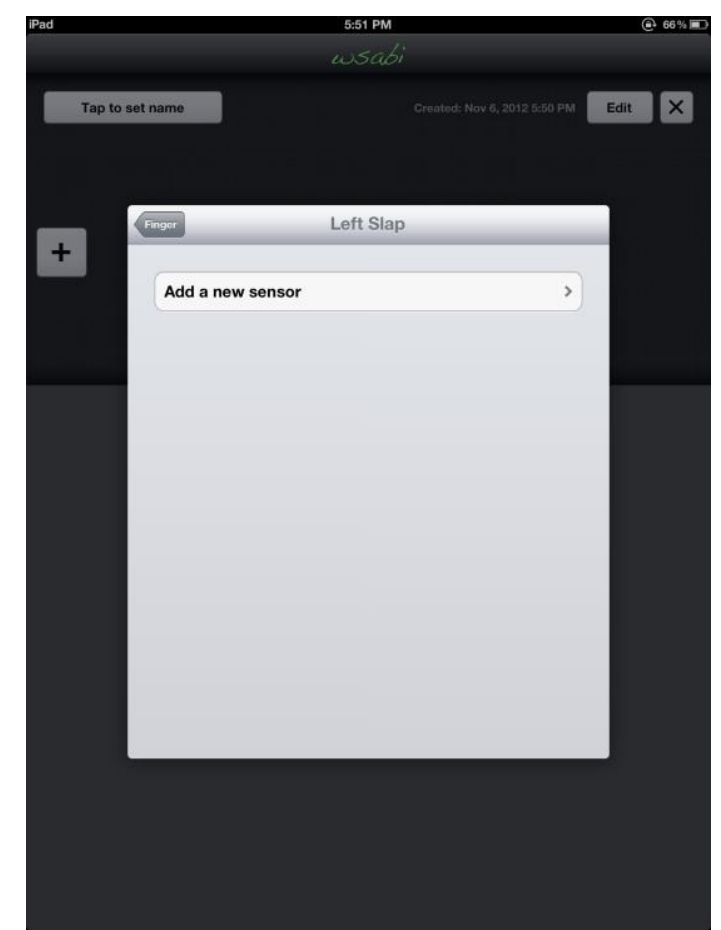

Figure 18. Third screen of sensor walkthrough: add a new sensor.

The fourth and final screen of the sensor walkthrough sequence had three subtasks (Fig. 19). Notice how the sensor name appears at the top of the walkthrough, and again the "back" button is informatively labeled.

1) Tap in the "Network address" text field to bring up the keyboard and enter a sensor's IP address. We used "test.me/finger" as the address, so that participants did not have to change onscreen keyboards to find the numbers that would be required in a typical IP address. After a network address is entered, the system automatically tries to connect to a sensor located at that address. While checking, the message "Checking" along with a moving activity symbol appears. When a sensor is found, a green checkmark appears with the message "Found a sensor at this address." If one is not found, the message "No sensor found at this address" would appear, along with a button labeled "Check again."

2) Tap in the "Name" text field to enter the name of the sensor. Note that it is not necessary to enter a sensor name per se; a network address is the only critical 
information to enable WSABI to communicate with a sensor. During testing we used "Finger Sensor" rather than "Suprema" as the sensor name. By using generic words, this allowed users to take advantage of the native iOS autocorrect features.

3) Tap the "Done" button in the upper right corner of the sensor walkthrough to finish the process and save entered information. Note that after a network address is entered, the Done button changes from gray (inactive) to blue (active).

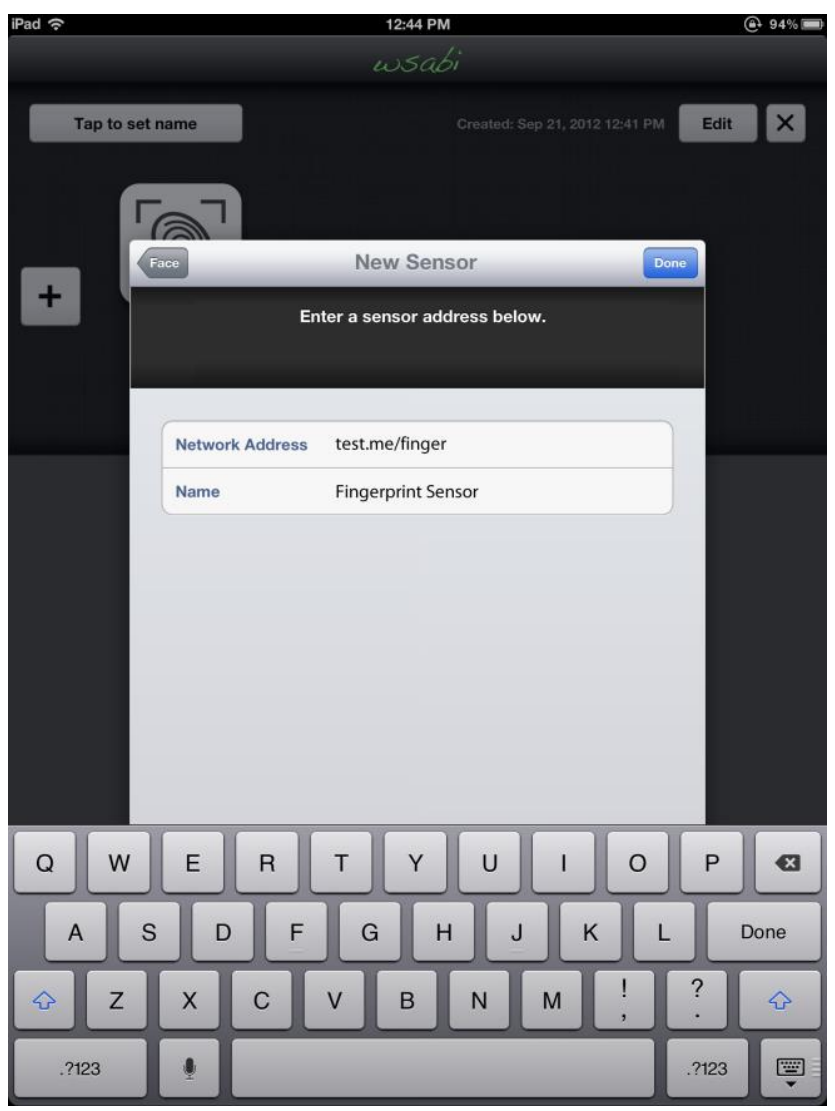

Figure 19. Fourth and final screen of sensor walkthrough: sensor information.

After stepping through setting up a left slap with the sensor walkthrough, a new person record is created. It contains a single fingerprint icon with the words "Left Slap" underneath (Fig. 20). 


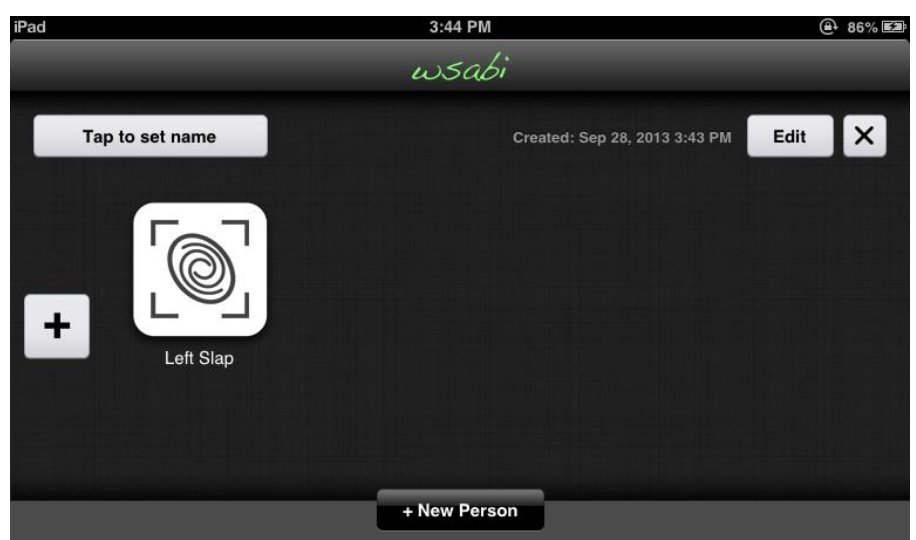

Figure 20. Active person record, after configuring a new sensor to capture a left slap.

Setting up a right slap was the next manager task. To add a new biometric, start with a tap on the square plus button on the left side of the active person record (previous Fig. 20). This brings up the sensor walkthrough. As before, tap the "Finger" menu button to select capture type/modality on the first screen of the sensor walkthrough (previous Fig. 17). On the second sensor walkthrough screen, tap the "Right Slap" button; note that it is immediately below the previously selected "Left Slap" button (previous Fig. 17). On the third screen, recently created/used sensors are listed under "Recent sensors" at the top of the screen. Therefore, instead of tapping the "Add a new sensor" button (previous Fig. 18 ), it is only necessary to tap the button with the name of the desired sensor, "Finger sensor" in this case (Fig. 21). While multiple sensors are shown for illustrative purposes in the screenshot (Fig. 21), during testing only one recent sensor appeared in the list.

\begin{tabular}{|l|}
\hline Finger \\
\hline $\begin{array}{l}\text { Recent sensors } \\
\begin{array}{l}\text { Finger sensor } \\
\text { test.me/finger }\end{array}\end{array}$ \\
\hline $\begin{array}{l}\text { Finger1 } \\
\text { wsbd2/finger1 }\end{array}$ \\
\hline $\begin{array}{l}\text { Finger2 } \\
\text { wsbd2/finger2 }\end{array}$ \\
\hline Add a new sensor
\end{tabular}

Figure 21. Recent sensors list on third screen of sensor walkthrough. 
On the fourth screen of the sensor walkthrough, the network address and sensor name fields are auto-populated with the information previously entered for the selected sensor. Again, the system would automatically check for a sensor at that address (Fig. 22). Note that the back button (Fig. 22) now says "Right Slap" whereas during setup of a left slap (previous Fig. 19), it said "Left Slap".

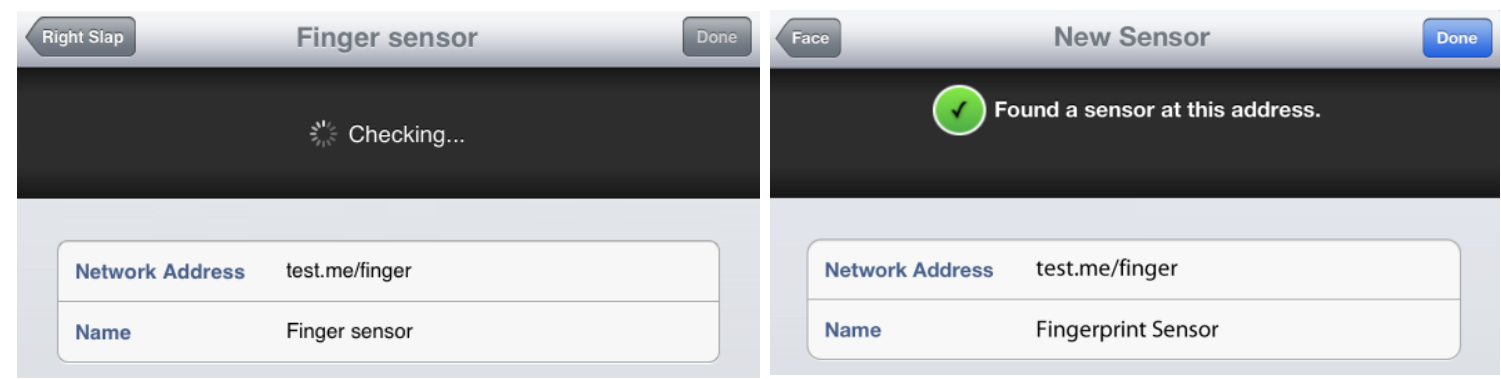

Figure 22. Partial fourth screen of sensor walkthrough during right slap setup.

A green checkmark and the message "Found a sensor at this address" then replace the "Checking" message, and a user must tap on the "Done" button to save settings and exit the sensor walkthrough (Fig. 22). A second fingerprint icon, with the words "Right Slap" underneath, is then added to the same person record created during the left slap setup task. The new icon is added to the end of the active person record (Fig. 23). All other buttons remain in the same location; for all tasks involving adding a new biometric, the user is tapping the same target (the square plus button) in the same location to bring up the sensor walkthrough. 


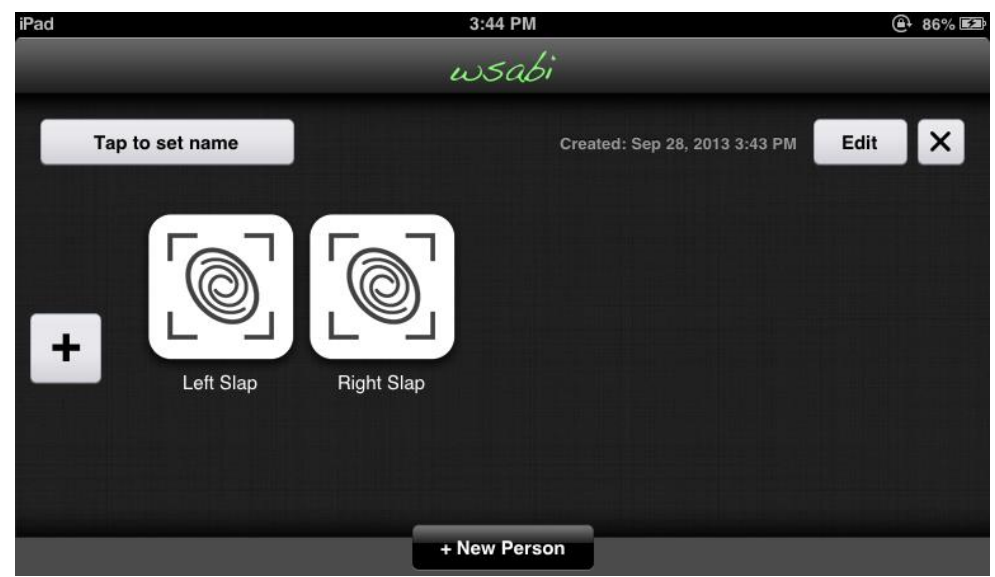

Figure 23. Active person record, after right slap setup.

After the right slap configuration task, the next manager task was to set up a face image. To add a new biometric, as before, tap the square plus button on the active person record to enter the sensor walkthrough. On the first screen of the sensor walkthrough, tap the Face button to select the face modality. The Face button is immediately below the Finger button; the most frequently used biometric modalities are always at the top of the list. On the second screen, tap the face button to select a "regular" two-dimensional (2D) face image rather than a three-dimensional (3D) one (Fig. 24).

\begin{tabular}{|c|c|}
\hline Capture type & Cancel \\
\hline Face & $>$ \\
\hline Face (3D) & $>$ \\
\hline
\end{tabular}

Figure 24. Face submodality selection on second screen of sensor walkthrough.

On the third screen, tap the "Add a new sensor" button. Had any face sensors been previously configured, they would have been listed under "Recent sensors" on this screen. Only sensors relevant for the chosen modality and submodality are displayed to the user in the sensor walkthrough. On the fourth screen of the walkthrough, enter "test.me/face" in the network address field, and "Face sensor" in the sensor name field. 
Again, wait for the green checkmark and "Found a sensor at this address" message to appear, then tap the "Done" button. A face icon with the word "Face" underneath is then added to the same person record, at the end of the row (Fig. 25).

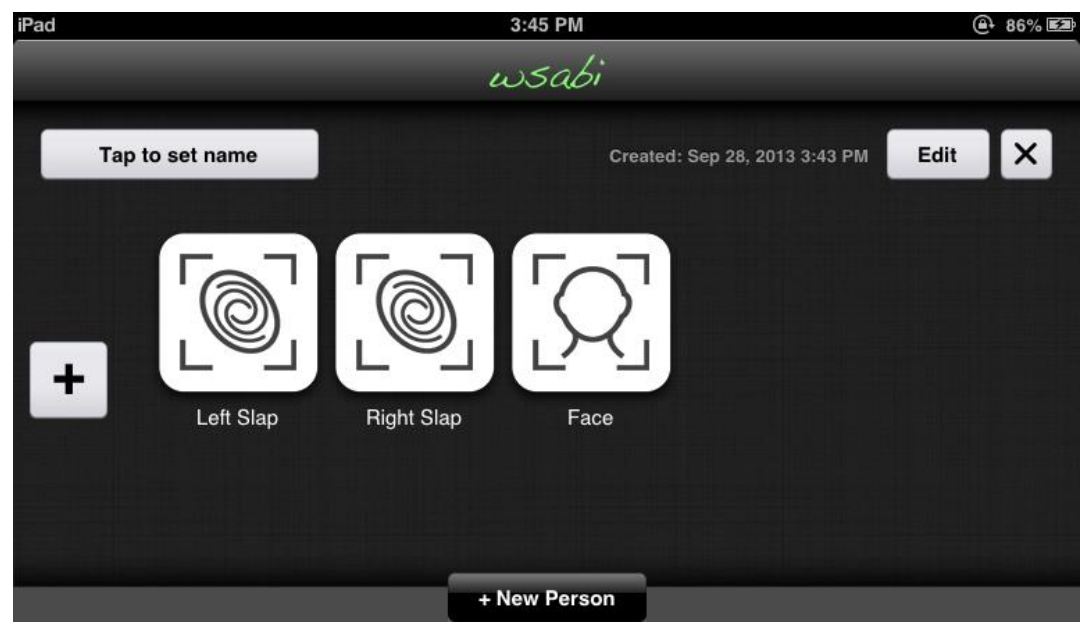

Figure 25. Active person record, after configuration of face capture.

We then asked participants to organize the screen so that the face was first. All biometric data icons within a person record can be rearranged using the "drag and drop" method common across most touchscreen devices. Simply press and hold the face icon until it wriggles, then drag it to the left of the two fingerprint icons. Release it (drop it) and the other icons automatically move to the right to accommodate it. Since biometrics can be captured in any order, such rearrangement was not actually necessary; we merely wanted to test the direct manipulation affordances of WSABI's biometric icons. After setting up a left slap, right slap, and face, then rearranging the biometric icons, the basic employee template creation was completed.

\section{Guard template}

The next manager task was to create a guard template, which is identical to the basic employee, with the addition of a thumbs slap. There are multiple ways to do this, depending on whether one wishes to create an entirely new template or merely modify 
the existing one. To create a new template, tap the "New Person" button. This creates a new person record identical to the previous one, set up to capture face, left slap, and right slap (Fig. 26). The new person record is created below the existing one(s), and automatically becomes the active row; all other records are grayed out. As before, to add to the current template, simply tap the plus button to bring up the sensor walkthrough and configure a thumbs slap. Select "Finger" on the first screen; select "Thumbs Slap" on the second screen; select "Finger sensor" under recent sensors on the third screen; wait for the "Found a sensor at this address" message and tap "Done" on the fourth screen. If someone chose to modify the existing template rather than create a new one, the only difference in procedure would have been an omitted tap on the "New Person" button.

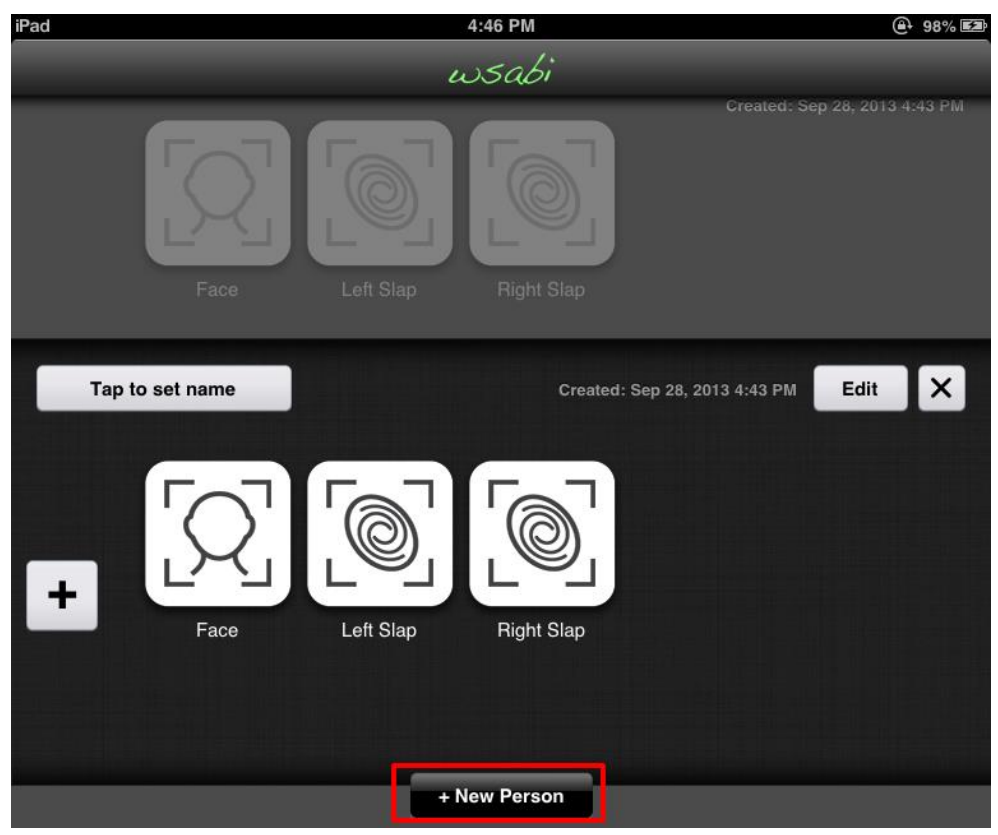

Figure 26. Multiple records.

\section{Operator Tasks}

As in Study 1, the researcher played the role of different employees, and the participant played the role of an operator responsible for taking employees' biometrics. Before starting the operator tasks, the researcher handed the participant a different iPad with a 
single person record on it; that record contained no data, but was configured to capture a left slap, face, and right slap.

\section{“Alice” tasks}

First, the researcher again played the role of Alice. As in Study 1, participants were to capture a left slap; clear and retake the left slap; capture a face image; start the right slap but interrupt to retake the face; return to finish the right slap; annotate the left slap due to a bandaid making the left index finger unprintable. An entirely new task (biographic information entry) was added to the beginning of the original Alice task sequence in order to test the new biographic information fields.

\section{Biographical information entry}

For the new biographical information task, the researcher pulled a badge from her NIST badge holder and handed it to the participant. Along with the researcher's picture, the badge contained only three pieces of biographical information, in the following order (with similar alignment and spacing):

Name: Alice.

DOB: January 1, 1981.

Gender: Female.

To enter biographical information, tap on the button labeled "Tap to set name." Then tap on the First Name field to bring up the onscreen keyboard and type Alice. Next, tap on the DOB button, scroll to select the appropriate date, and tap Done when finished. Tap the Gender button, scroll to select, and tap Done when finished. The DOB and gender biographical information wheels, and the completed fields, are shown in Fig. 27. 

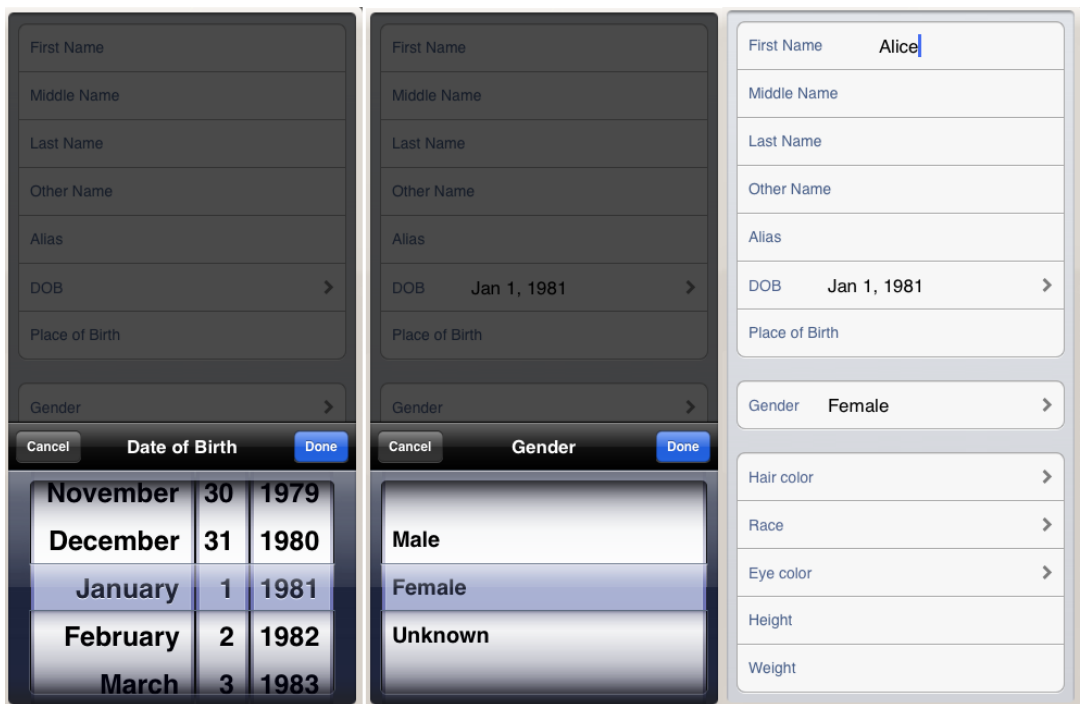

Figure 27. Biographical information task completed.

\section{Capturing}

To capture a left slap, tap the small left slap icon to bring up the large capture card, then tap the animated hand on the capture card to start the capture (previous Fig. 12). The presenter (in this case, the researcher) places her left hand upon the sensor, then the slap image appears in the capture card on the iPad. A small thumbnail version of the image also appears in the previously empty left slap icon. During any delay while the tablet is communicating with the sensor, a "Waiting for sensor" message appears in the capture card (Fig. 28).

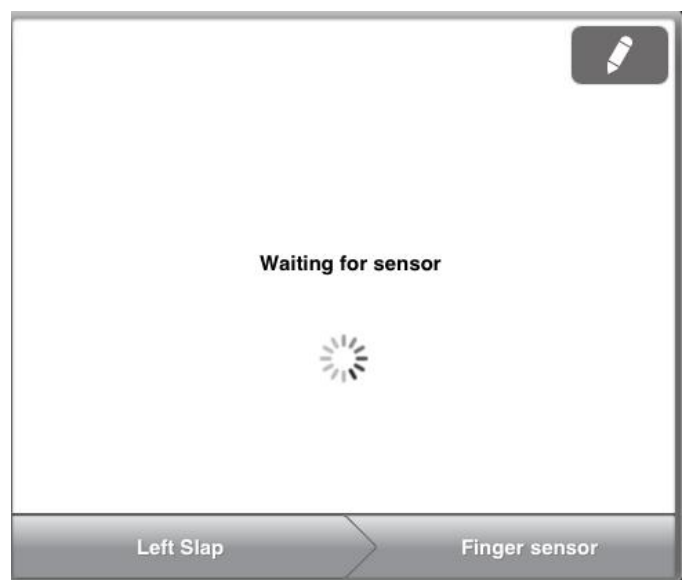

Figure 28. "Waiting for sensor" message during capture. 


\section{Clearing and retaking}

To clear (or annotate) the image, tap the pencil edit button in the upper right corner of the capture card. Options slide up within the capture card: Clear this image, Annotate, or Cancel. Tap the "Clear this image" button. Since clearing is a destructive action, a confirmation is required. Tap the "Clear" button to confirm. (Clearing and confirmation both shown in Fig. 29.)
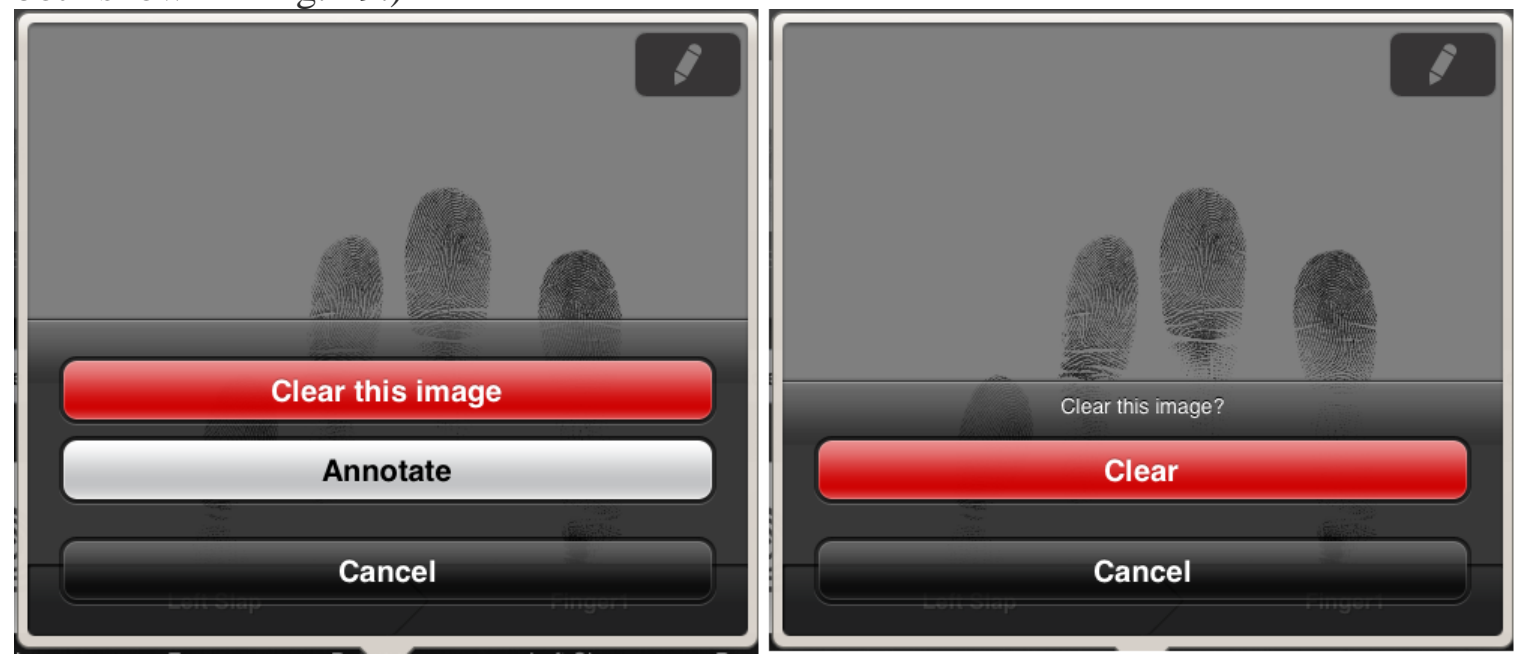

Figure 29. Clearing an image.

After an image is cleared, the animated hand reappears within the capture card and the system is ready to retake an image. Since the process for retaking/taking the other biometrics is identical, additional screenshots are not shown here for the right slap and face capture tasks.

\section{Annotating}

To annotate the left slap indicating Alice's index finger could not be acquired due to her bandaid, first tap on the small left slap icon to bring up the capture card. Next, tap the pencil edit button, tap the "Annotate" button, and the capture card flips over. We simplified the annotation options from Study 1, replacing the unprintable versus amputated options with a graphical symbol (Fig. 30). Tap on any or all of the fingers listed to indicate an issue printing that finger; tap again to remove the symbol. Enter any 
notes, such as "bandaid", in the free-form notes field. Tap the "Done" button to flip the capture card back over. On the front side of the capture card, a blue annotation badge has now appeared on the pencil edit button. A matching blue annotation badge has also appeared on the small left slap thumbnail in the person row.

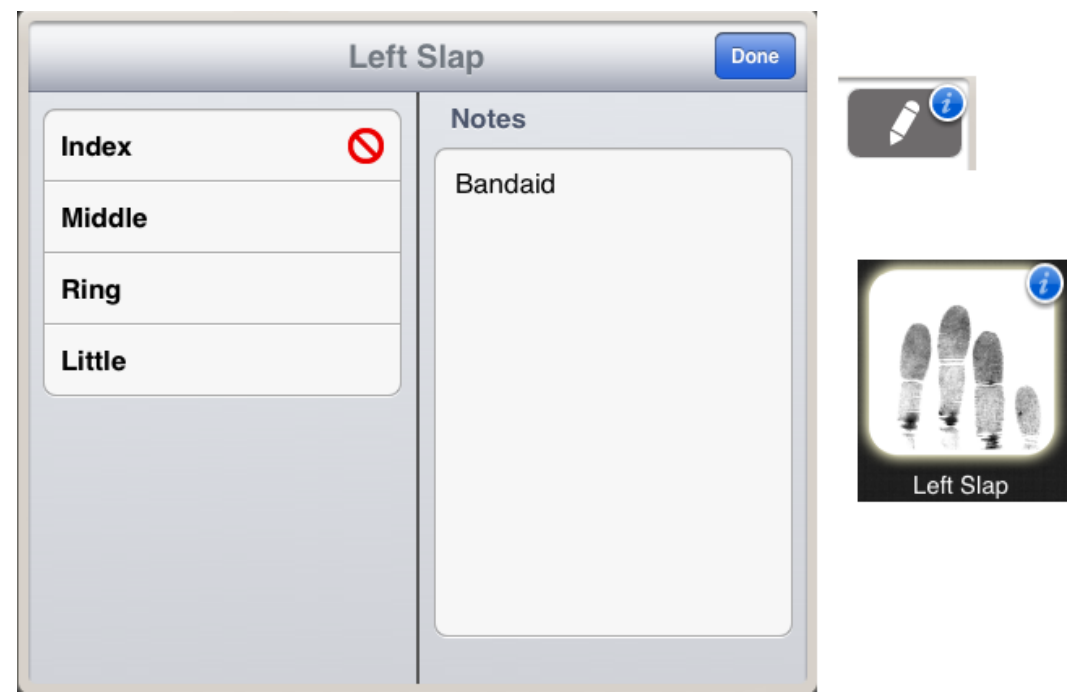

Figure 30. Annotations.

"Bob" tasks

As in Study 1, after playing the role of Alice, the researcher played the role of Bob. As before, participants were to create a new record, then capture a left slap, face image, and right slap. During the right slap capture, "Bob" said he was not feeling well and could not continue. In Study 1, this "not feeling well" scenario was designed to test the explicit capture cancel button. In Study 2, there was no longer an explicit capture cancel button (simply tap outside the popover to dismiss it, as with most touchscreen popovers). However, we kept the scenario, because we were interested in seeing whether people would use either of the two new free-form notes fields, one on the back of the capture card, and one at the bottom of the biographical information field. The only new task during the Bob scenario was the naming task. The participants were told "So we can tell the two employees apart, please indicate that this is Bob." First name was the only 
biographical information entered for Bob.

"John" tasks

The researcher then played the role of another employee, John. These tasks were entirely new, as there was no John scenario in Study 1. In this scenario, participants were told John was a current employee who just needs a picture for a replacement badge. They were to capture his face image, after which they were told "His fingerprints are already on file, so please remove the fingerprints from his row." This was designed to test the new delete-item functionality. (Recall that in the original WSABI, one could only delete an entire collection; there was no option to delete individual biometric items.)

\section{Deleting an item}

To delete an item, tap the Edit button at the top of the active record. All items are now in an editable state, with a red $\mathrm{X}$ over the center of each item; in this editable mode, the Edit button is changed to a Done button. To delete an individual item, tap on the red $\mathrm{X}$ in its center, then tap the "Confirm Delete Item" button. The sequence of delete-item actions is shown in Fig. 31, with Edit/Done buttons circled. Note that the red Xs are located in the center of the icons rather than in their upper right corners. While the latter position is common when icons are in an editable state on the iOS home screen, we did not want to give the impression that items were rearrangeable in this case. We intentionally removed the "drag and drop" affordances by placing the Xs in the center of the icons, exactly where people would be most likely to press-and-hold to execute a drag. 

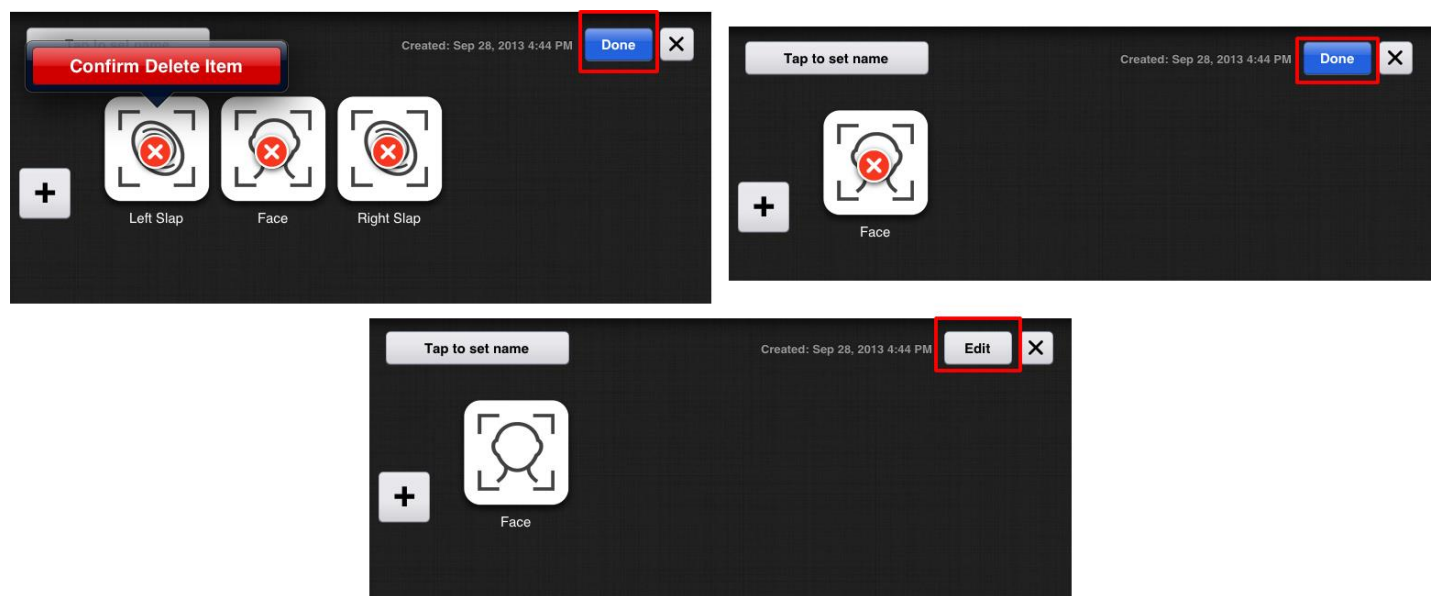

Figure 31. Delete-item task, editable (top) and non-editable (bottom) states.

\section{Deleting a record}

To test the delete-record functionality, participants were told that "Your boss calls and says John just retired, so please remove his row entirely." To delete a person record, tap the $\mathrm{X}$ button in the upper right corner of the active record, then tap "Delete this person." The entire record is covered with a red highlight, over which the buttons "Cancel” and "Confirm Delete Person" appear (Fig. 32). Tap "Confirm Delete Person” to delete the entire record.
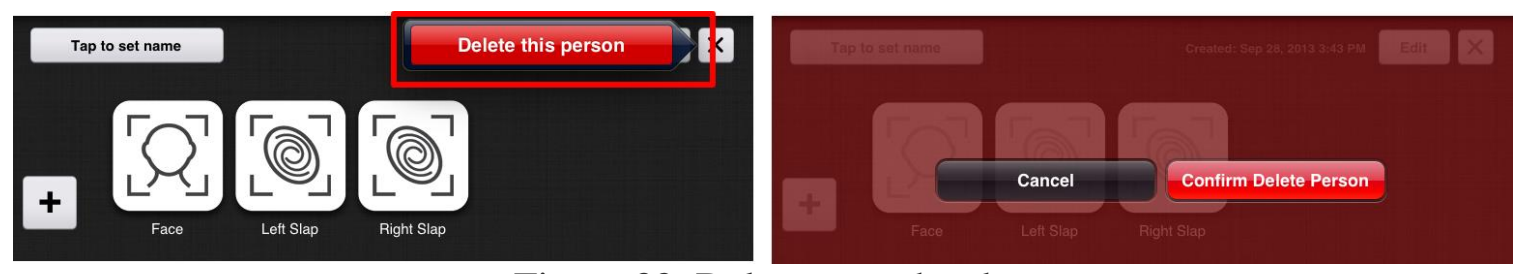

Figure 32. Delete-record task.

As with the clearing of an image, deleting a biometric item or an entire person record are destructive actions, hence an explicit confirmation is required for all. Note that technically the delete-person action actually requires a second confirmation, since of the various delete functions, it could result in the greater amount of lost data if done accidentally. 


\author{
Results \\ Manager Tasks
}

\title{
Basic new employee template
}

Left slap setup

All participants advanced successfully through selecting the finger modality, left slap submodality, and selecting "add a new sensor" to make it to the sensor information screen of the sensor walkthrough. There was one participant who hesitated over selecting the finger modality for the capture type, saying "If it's not finger, I would try Other". This makes perfect sense for someone with little background in biometrics; we asked for a left slap, and left slap is not displayed as an option until the modality selected is finger.

Interestingly, the greatest difficulty users had during the left slap configuration task was typing in the sensor name and network address. Recall that on the placard with the sensor information, the sensor name was capitalized as "Finger Sensor." When typing in an empty text field, the first word is automatically capitalized with the default iOS keyboard settings, but subsequent words are not (unless they follow a period and a space). Therefore, the capitalized second word in the sensor name was slightly problematic for users. Almost all participants asked whether the capitalization mattered, to which the researcher replied no, it is not case sensitive. Some participants struggled to capitalize the second word in the sensor name before asking about it, others asked immediately. For those that attempted capitalization, their difficulty was in finding the shift key on the onscreen keyboard. Participants also had minor difficulty finding the forward slash symbol while typing in the network address. This was not surprising given that text entry on touchscreen keyboards is notoriously problematic, both slow and errorprone. Text entry metrics are a popular research area in HCI; research that began with many years of transcription studies conducted with typewriters and desktop computers has now moved into the mobile domain. 
Aside from the text entry itself, participants did not have difficulty mapping the information from the sensor placard into the appropriate fields in the sensor walkthrough. Only one participant did not enter a name for the finger sensor, but as previously mentioned, the network address is the only critical sensor information. All participants successfully entered the network addresses, which is extremely important, as without a valid network address, WSABI cannot communicate with and receive images from a sensor.

\section{Right slap setup}

After configuring the first biometric (left slap), setting up any new biometrics required pressing the square plus button to bring up the sensor walkthrough. During the debriefing, one participant expressed that they weren't initially sure about using "the edit button versus the plus mark to add, because sometimes you can add from within edit," and that they were "trying to decide between new person, plus, and edit." Despite initial uncertainty, the participant was able to complete the task, as were all other participants.

Once in the sensor walkthrough, picking "Finger sensor" from the list of recent sensors meant that the associated information was autofilled, so participants did not have to retype the network address and sensor name during the right slap task. For the one participant who did not name the finger sensor during the preceding left slap task, the sensor appeared with its network address rather than name on the list of recent sensors. This participant noted in the debrief "I thought that when adding the sensor, you can find it by the name. I don't think it picked it up, so it threw me off." It seems likely that they were expecting entering the network address to automatically pull up the corresponding sensor name within the application. 


\section{Face setup}

One participant asked "Is this for the same person?" before starting the task. As with the right slap task, setting up the face biometric required pressing the square plus button to bring up the sensor walkthrough. A different participant tapped the new person button two times before trying the plus button. Across participants, there was some uncertainty regarding whether to select Face or 3D Face as the submodality during this task. When participants asked which to choose, the researcher explained that the sensor couldn't take 3D images, at which point all participants correctly selected Face rather than 3D Face. As with the initial left slap task, adding the network address and name for the new sensor gave rise to the same text entry issues previously seen.

\section{Rearranging icons}

Recall that in order to drag and drop icons, one must press-and-hold one until they are all in a moveable state (i.e., until they start wriggling). While markedly improved over the press-and-hold delay issues participants experienced when trying to rearrange workflow items in the original WSABI interface, the new delay was not completely without issue. All participants attempted the drag and drop appropriately; three participants did not initially press-and-hold long enough before attempting to move the face icon. As before, the researcher reassured them that they were doing it exactly right, and explained the required delay. With this explanation, they were able to successfully rearrange the icons. 


\section{Guard template}

Thumbs setup

The thumbs setup task was particularly interesting because there were multiple ways to accomplish it, either of which were correct. Participants could add the thumbprints to the existing record or create a new person record, and they could add a thumbs slap or add a left and right thumb instead.

Multiple participants were unsure whether to add the new thumb biometric to the existing person record (i.e., the basic employee template) or create a new record for the guard template. Before starting this task, one participant (the same participant who previously asked "Is this for the same person?" during face setup) asked "Can I add onto this one or do I have to do a totally new one?", to which the researcher replied that either method was fine. A different participant hesitated over the New Person button, and during debriefing commented that "This isn't a person, this is a template for all people, that's why I didn't click the new person button. I was trying to decide between new person, plus, and edit." Despite this uncertainty over the New Person button, the same participant seemed pleased with its functionality, commenting that "When I added a new person during the template it was nice that it did everything for me."

Note that the script merely said "[guards] also need thumbs recorded", at which point the researcher would demonstrate a thumbs slap. Three participants extrapolated from the previously defined term "left slap" and its associated demonstration, and added a "thumbs slap" here; two of these explicitly asked whether they should select thumbs slap upon seeing the submodality options. Rather than adding a thumbs slap, the remaining four participants added the left thumb, followed by the right thumb. Adding the two thumbs separately was equally as effective as adding a thumbs slap, albeit slightly less efficient, as stepping through the walkthrough a second time required five extra taps. 


\section{Operator Tasks}

\section{Biographical information entry}

All participants successfully completed the biographical information entry task, typing the first name Alice, and using the date and gender selector "wheels" to enter her DOB and gender (previous Fig. 27). In contrast to typing the network address and sensor name during the preceding manager tasks, participants did not have difficulty typing "Alice," since it was auto-capitalized and did not require any special symbols. Participant strategy with the year selector wheel varied in terms of the magnitude of their scrolling/flicking gestures. Some participants made small, conservative scrolling gestures with the year selector wheel, only advancing it a few years at a time; others used larger scrolling gestures with a more aggressive "overshoot then correct" strategy. One participant commented that "The spinner is easier" [i.e., the biographical wheels are easier than typing], while another noted that they were "fun" to use. There were no issues with the gender selector wheel, although several participants seemed to find the "unknown" gender option rather humorous.

Of particular interest were participant comments regarding saving the biographical information they just entered. There was actually no need to explicitly save any work. There was no "save" or "done" button in the biographical information popover; to dismiss the popover, simply tap anywhere outside it. Data is automatically saved as soon as it is entered (or captured). Based on Study 1 findings, information about saving was added to the script, i.e., "Don't worry about saving your work; the iPad does that for you automatically." However, participants still seemed concerned about saving, with several asking how to save during this task. Debriefing comments such as "Done button might be nice for the bio info." further support these observations. 


\section{Multi-modal capture}

As in Study 1, all participants successfully performed the multi-modal biometric acquisition tasks. The common capture cards again worked well across modalities. As before, there were several participants who instructed the researcher (in the role of Alice) to place her hand on the sensor, and seemed to expect the image to automatically appear on the iPad. As before, when no image appeared, participants realized an action was necessary on their part, after which they correctly tapped the animated hand on the capture card. One participant commented that they were initially "confused as to who was supposed to touch it", thinking the animated hand indicated an action was required by the presenter rather than the operator. In other words, they initially thought that the animated hand meant the presenter was supposed to place her hand upon the sensor. However, this did not prevent task completion, and the same participant commented that "Once I understood I was to touch it, it was easy."

During debriefs, there were several questions regarding the "Waiting for sensor" message. For example, one participant asked "Is it waiting saying now you should put your hand on it, or is it already reading?" and another asked "Does your hand have to be on it first and then press the button, or is it ok at the same time?". Participant uncertainty regarding the readiness of the biometric device highlights the need for sensor affordances, as described in prior biometric usability work (Theofanos, Stanton, and Wolfson, 2008). While sensor interaction was out of scope for the current studies (NIST does not test products), real-world deployments of WS-BD systems should consider such issues. 


\section{Retaking and annotating}

As in Study 1, participants were successful at clearing, retaking, and annotating images. The pencil edit button again worked well, and with the simplified annotation options, participants were no longer uncertain whether to select "unprintable" versus "amputated". The free-form notes field worked extremely well; all participants used it in some fashion (notes varied from simply "bandaid" to "Print could not be read properly"). In contrast to the notes field on the back of the capture card, participants did not use the free-form notes field in the biographical information popover. This may have been because it was at the bottom of the biographical information list and not visible without scrolling. Unfortunately, it was not possible for all biographical information to be simultaneously visible in the current interface, in order to accommodate the onscreen keyboard necessary for text entry.

\section{Deleting items and records}

The modal Edit/Done button again posed difficulty, albeit for different reasons and to a much lesser degree than in Study 1. In Study 2, the editable state indicators were significantly more visually salient than they were in Study 1 (Fig. 33). Furthermore, a tap anywhere other than on a red X or blue Done button would automatically change the collection back into its default non-editable state in Study 2; in Study 1, a collection remained in an editable state unless the Done button was tapped. However, one cannot fully compare Edit modes across studies, since the type of deletion enabled during Edit

mode differed. In Study 1, Edit only allowed deletion of an entire collection, whereas in Study 2, it allowed deletion of individual items within a collection. 

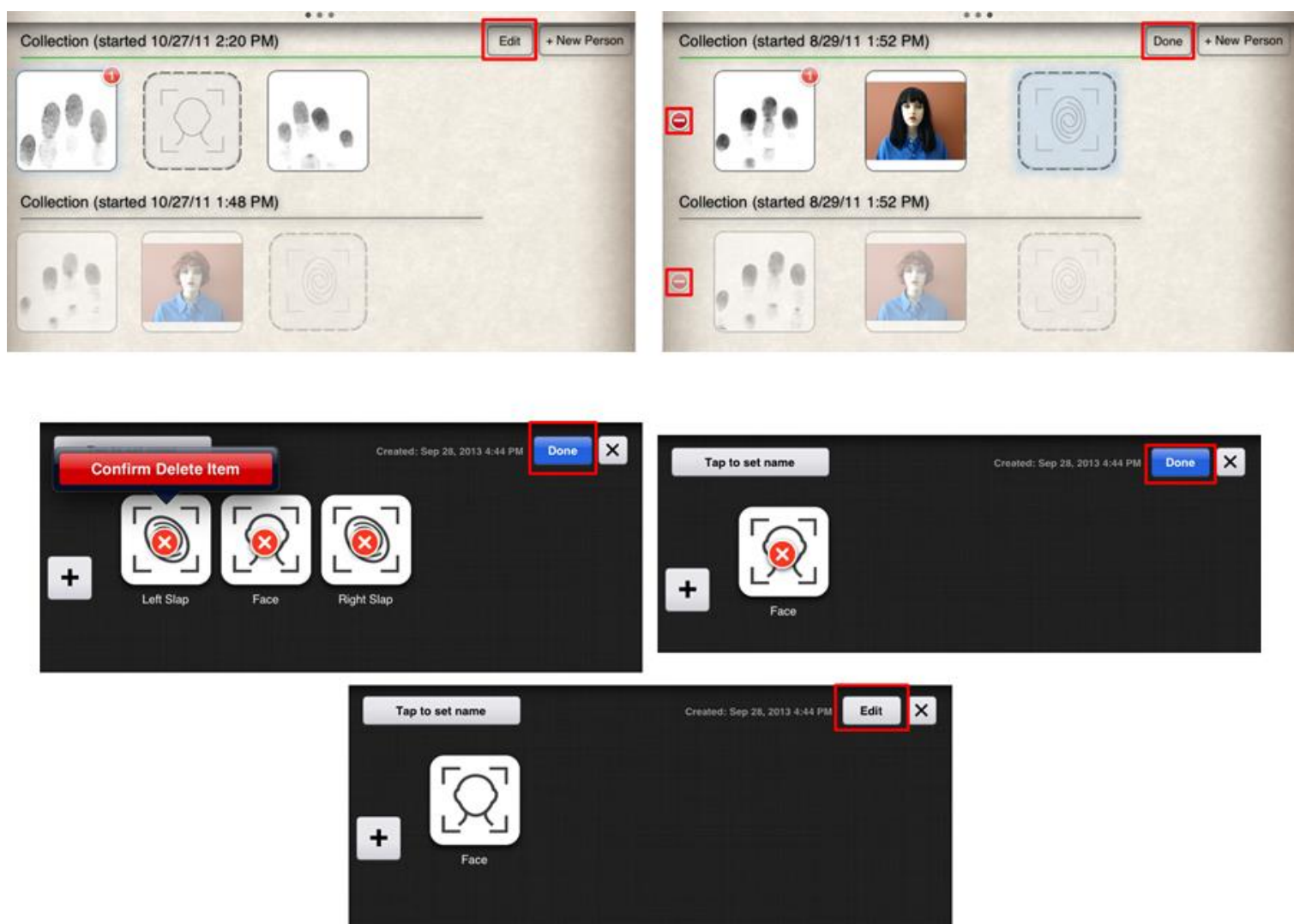

Figure 33. Edit/Done buttons and modal states for collection-deletion in Study 1 (top) versus item-deletion in Study 2 (bottom).

The delete-item and delete-record confirmations seemed superfluous and/or confusing to multiple participants. For example, one commented "the confusing part was the deleting, I had to push the delete button again to delete." While it would certainly be possible to reduce the number of actions required to delete data, until an undo option is implemented, it seems more prudent to err on the side of caution where such destructive actions are concerned.

Across manager and operator tasks

\section{Saving}

There were two separate saving issues: one in the biographical information popover (operator tasks), and one in the sensor walkthrough (manager tasks). 
Two participants explicitly mentioned looking for a Done button on the biometric information popover. Participants searching for a "Done" button in the biographical information task is indicative of a general conceptual difference between desktop and mobile computing regarding "saving." In the traditional desktop paradigm, users are frequently required (and prompted) to explicitly "save" their work. In the mobile computing paradigm, saving is more commonly implicit and handled automatically by the system.

Almost the reverse issue occurred in the sensor walkthrough, where a tap on the Done button was required to save the sensor information and dismiss the walkthrough. In this case, the button was present and all participants clearly saw it. However, in two instances, a missed tap on the Done button resulted in dismissing the walkthrough prematurely; this is quite a severe error, as the sensor information just entered was dismissed as well. The issue arose due to small variations in human motor movement accuracy; the most significant issue with the sensor walkthrough was caused by one of the smallest buttons, the "Done" button on the final screen of the walkthrough.

\section{DISCUSSION AND CONCLUSIONS}

The benefits of consistent procedures and interface elements cannot be overemphasized. We made numerous significant changes in the WSABI interface from Study 1 to Study 2, ranging from functional to merely aesthetic. Yet by keeping core features and affordances of the biometric capture cards consistent, novice participants were able to successfully complete multimodal capture in both studies, without training. In contrast, current software training can take four to six hours per device even for experienced operators (R. Bowlen, FBI, personal communication, November 30, 2012). The implications of our results are far-reaching. Consider the enormous amount of money and time currently spent on training and retraining operators to use different biometric devices. Consider the amount of money and time currently spent on updating biometric 
device drivers and software, on addressing interoperability issues between devices and clients, on creating a custom "middleman" software solution, etc. Now imagine the savings that would be realized by capitalizing on the human cognitive and perceptualmotor benefits that accompany consistent procedures and interfaces. Although biometric sensors and mobile computing devices will continue to evolve, that should not necessarily require costly user retraining each time technology changes.

Based on the results of our two small formative usability studies, this seems more than feasible. To support reduced user training requirements, there are several general guidelines we recommend following when designing touchscreen interfaces for multimodal biometric capture (below). While it seems obvious that general HCI principles should apply to biometric capture interfaces, it is worth emphasizing that while these may be ingrained principles for cognitive scientists and usability professionals, they are not necessarily so for software developers.

\section{Design recommendations}

- Design biometric acquisition software to be user- rather than sensor-centric.

- Keep the core capture primitives constant. Taking, clearing, retaking, and annotating biometric data are core functions of the capture process; these basic capture tasks should require similar actions regardless of biometric modality or sensor.

- Be consistent. E.g., consistent buttons, consistent locations, consistent procedural steps, consistent language.

- Leverage direct manipulation features of touchscreens. Have editing functions near the objects in question, e.g., the pencil edit button is on the image it will annotate.

- Capitalize on affordances. For example, objects that look like buttons will encourage people to tap them (e.g., the breadcrumb bar buttons at the bottom of the capture cards) 
- Use internationally tested, standardized symbols where possible (e.g., the small biometric modality icons)

- Use modal features sparingly and carefully (e.g., Edit/Done mode)

- Be aware of differences between desktop and mobile computing paradigms. Think carefully before adding features like Done/Save buttons simply to meet user desktop expectations. While it may match their desktop paradigm, it is at odds with-and unnecessary for - the mobile computing paradigm. Therefore, adding it would add a superfluous step and make the task less efficient, especially when scaled up to numerous enrollments. Don't assume that what works well in a desktop application will necessarily work well on a mobile device without requiring modification (e.g., the workflow concept and button). Desktop versus mobile are two different computing paradigms.

- Provide useful, informative feedback to the user regarding system status, and provide human-readable error messages with recovery options (e.g., the "try again button" in sensor walkthrough, or the "waiting for sensor" message on a capture card). Furthermore, because error states are often fleeting and can be extremely difficult to reproduce, expect that there will be fringe cases that simply won't appear during usability testing. It is critical that software developers are knowledgeable about the requirements for user-friendly, meaningful system feedback.

- Don't assume that users will know features of the native operating system, even if they have experience with the device (e.g., the press-and-hold timing; the onscreen keyboard). 


\section{FUTURE DIRECTIONS}

Both the WS-BD protocol and the WSABI reference implementation already support transfer of static images from any compliant sensor (i.e., any sensor that conforms to the WS-BD specification). Recent work on both now allows video streaming. Adding such live preview functionality is highly beneficial, enabling operators to see images before actually capturing them, which is more analogous to the alreadyfamiliar process of taking a picture with a digital camera or smartphone. Additional recent work has also incorporated new passport reader technology, so that using a sensor to scan a driver's license or passport automatically transfers appropriate information to the client device. These newer features and functionality should undergo formal usability testing as well.

As previously mentioned, WS-BD is device and operating-system agnostic. Conceptually, WSABI should be as well. To fully test this, it should be implemented on additional platforms, such as Android tablets. It should also be implemented on smaller devices, such as smartphones and blackberries. To do this will require some redesign, such as making better use of screen real estate by reducing the empty spaces between icons and buttons, or by making use of disclosure triangles to show/hide information. Such redesign should not require retraining users if WSABI's common capture primitives are implemented consistently, but this must be verified experimentally via formal usability testing. If WSABI's existing conceptual model ports well across mobile device sizes, then we will have truly achieved a flexible, extensible, consistently designed reference implementation that exercises all relevant features of the WS-BD specification. 
We intentionally designed WSABI for, and tested with, novice users. This was appropriate given our goal of exercising the WS-BD protocol; since WS-BD only deals with communication between clients and sensors, we did not implement functionality to support certain real-world operator tasks that come before or after biometric capture. For example, WSABI does not have built-in support for submission (i.e., an automated process for sending images in standard format to query a fingerprint database). Researchers and institutions who wish to use the WSABI reference implementation as a starting point for larger, real-world biometric systems will need to extend and customize our open-source software for their specific needs. Most likely this would include addressing issues such as submission and scalability. For example, WSABI is not particularly efficient for large numbers of records in its current implementation; too much scrolling would be required. To address scaling issues, one might implement various searching and sorting functions; with such additional functionality, a user could sort by name, by date, by age, etc., or search for a specific record.

Future WSABI extensions should add new functionality designed for advanced users. For new pre-capture functionality, one could add import capabilities that would allow an operator to import a database of biographical information for a number of new employees simultaneously. Combine this with a script that automates new record creation and pulls from the imported database to auto-populate the biographical information fields of numerous new records simultaneously. Then during enrollment, an operator would only have to complete the biometric capture(s) rather than performing any template setup or biographical entry tasks. 
Mobile text entry is notoriously slow and error-prone, so entering complete biographical information would be agonizingly tedious with an onscreen keyboard. The smaller the client device, the more problematic touchscreen-based text entry becomes. To at least save users the difficulty of capitalizing the first letter of each word in a text field, future work should utilize the iOS option to title case words automatically. For instances where heavy text entry may be still be required, a full (physical) QWERTY keyboard could be made available to operators; most popular tablets support connected and wireless keyboards. As an alternative, the feasibility of implementing dictation capabilities to capture voice notes should be investigated; most smartphones already have native recording applications, some of which automatically reduce ambient noise.

Additional advanced features might include making a list of all previously used sensors available simultaneously. In the current WSABI implementation, only sensors relevant for the currently selected biometric modality are shown, but expert users may want to view all sensors regardless of which modality/modalities they support. For experienced users, the fingerprint icon should not be angled as it was in Study 2. It should be reverted back to its original, non-tilted orientation, as expert users may be bothered by the fact that the angled orientation is at odds with the ISO standard on international biometric symbols. The annotation options may also need to revert to their original form, which enabled users to mark individual fingers as unprintable versus amputated; for expert users, supporting the Electronic Biometric Transmission Specification (EBTS) may be important. 
Regardless of any such minor modifications to WSABI, the critical capture primitives should remain roughly unchanged in future work. Using capture cards, biometric icons, and annotation features consistently means that WSABI will always be poised for new modalities, such as ear and vein. Not only should WSABI undergo formal usability testing for new modalities, but also for newer iOS versions. For example, buttons in iOS 7 do not appear nearly as "button-like" as they do in iOS 6, which may negatively impact some of the affordances we worked so hard to create in our current WSABI implementation. Future work on such issues would benefit both our specific biometrics research as well as the larger HCI community. Finally, HCI researchers may wish to explore other gestures and determine which are universal across touchscreens and which are idiosyncratic to a particular device/platform. 


\section{ACKNOWLEDGEMENTS}

This research was sponsored by the National Institute of Standards and Technology (NIST), the Federal Bureau of Investigation (FBI), and the Department of Homeland Security (DHS), as part of the Biometric Web Services (BWS) project (http://bws.nist.gov). The authors gratefully acknowledge NIST BWS project team members Kevin Mangold and Karen Marshall, especially for Karen’s thoughtful review; Matthew Aronoff, a previous member of the NIST BWS team; Benjamin Long of NIST's Software and Systems Division for his thoughtful review; and all the participants who graciously donated their time to further this research. 


\section{REFERENCES}

Apple. (2014). iOS Human Interface Guidelines. Retrieved from https://developer.apple.com/library/ios/documentation/UserExperience/Conceptua 1/MobileHIG/Introduction/Introduction.html.

Apple. (2013). About Events in iOS. Retrieved from https://developer.apple.com/library/ios/DOCUMENTATION/EventHandling/Conc eptual/EventHandlingiPhoneOS/Introduction/Introduction.html

Aronoff, M., \& Micheals, R. J. The Biometric Client Configuration Language. National Institute of Standards and Technology Interagency Report (NIST IR) 7531. Retrieved from http://www.nist.gov/customcf/get_pdf.cfm?pub_id=33208

Dediu, H. (2012). Apple sold more iOS devices in 2011 than all the Macs it sold in 28 years. Retrieved from http://www.asymco.com/2012/02/16/ios-devices-in-2011vs-macs-sold-it-in-28-years/.

Greene, K. K., \& Tamborello, F. P. (2013). Initial ACT-R Extensions for User Modeling in the Mobile Touchscreen Domain. Proceedings of the $12^{\text {th }}$ International Conference on Cognitive Modeling. Carleton University, Ottawa, Canada.

Greene, K. K., Tamborello, F. P., \& Micheals, R. J. (2013). Computational Cognitive Modeling of Touch and Gesture on Mobile Multitouch Devices: Applications and Challenges for Existing Theory. Proceedings of the $15^{\text {th }}$ International Conference on Human-Computer Interaction. Las Vegas, NV.

Micheals, R. J., Mangold, K., Aronoff, M., Kwong, K., \& Marshall, K. Specification for WS-Biometric Devices (WS-BD) Version 1. National Institute of Standards and Technology (NIST) Special Publication 500-288.

Micheals, R. J., Stanton, B., Theofanos M., \& Orandi, S. (2006). A Taxonomy of Definitions for Usability Studies in Biometrics. National Institute of Standards and Technology Interagency Report (NIST IR) 7378.

National Science and Technology Council Subcommittee on Biometrics. (2006). National Biometric Challenge. Retrieved from http://www.biometrics.gov/Documents/biochallengedoc.pdf.

Orandi, S., \& McCabe, R. M. (2009). Mobile ID Device Best Practice Recommendation. National Institute of Standards and Technology (NIST) Special Publication 500280. Retrieved from http://www.nist.gov/itl/iad/ig/upload/MobileID-BPRS20090825-V100.pdf.

Theofanos, M., Stanton, B., \& Wolfson, C. A. (2008). Usability and Biometrics. Ensuring Successful Biometric Systems. National Institute of Standards and Technology Interagency Report (NIST IR). Retrieved from http://zing.ncsl.nist.gov/biousa/docs/Usability_and_Biometrics_final2.pdf. 


\section{APPENDICES}

\section{Appendix 1, Informed Consent Form}

\section{INFORMED CONSENT FORM}

\section{Project Title: "Usability Testing: Biometric Acquisition"}

Principal Investigator: Ross Micheals

Co-Principal Investigator: Matt Aronoff

\section{RESEARCH DESCRIPTION}

This study is being performed to determine how to improve the interface that we have developed for biometric acquisition. The research is funded by the Department of Homeland Security (DHS) and conducted by the National Institute of Standards and Technology (NIST). After providing demographic information (e.g., age, sex, education level), you will be asked to perform some electronic computer tasks related to biometrics. None of your biometric data will be recorded, and any demographic/survey data that you provide will be recorded only by a randomly assigned number and without any individual identifiers.

This process should take you no more than 30 minutes.

Confidentiality:

Your identity will be protected to the extent permitted by law, including the Freedom of Information Act. Members of the NIST Institutional Review Board (IRB), and appropriate NIST researchers, appropriate Department of Homeland Security employees, and other appropriate Federal employees may review the records of this study. The data will be used by NIST researchers to assess and improve this biometric acquisition interface. All of the data, including the demographic/survey data, will be recorded without identifiers. All of the data will be recorded only by a number, and will not be linked back to an individual in any way.

You are free to withdraw from the study, without penalty, at any time during the experiment. In total, we expect to have approximately 60 subjects complete the experiment.

There are no risks involved in participating in this study, nor are there any immediate benefits. The long-term benefits of this study to society should be improved biometric access

Contact Information:

For questions regarding this study, please contact Ross Micheals at (301) 975-3234, ross.micheals@nist.gov, or Matt Aronoff at (301)975-8778, matthew.aronoff@nist.gov. For questions regarding your rights as a human subject, please contact Richard R. Cavanagh, NIST IRB Chair, at (301)975-8301 or richard.cavanagh@nist.gov.

"I have read the above description of this research project. I have also spoken to the NIST researcher, who answered any questions I had about this project. I acknowledge that I have received a personal copy of this form. I agree to participate in this research and I understand that I may withdraw, without penalty, at any time. I am a NIST employee acting in the course of my official duties with my management's approval."

Signature:

Date:

NIST Researcher

Signature of NIST Researcher

Date:

Witness:

Witness Signature: Date: 


\section{Appendix 2, Demographic Questionnaire}

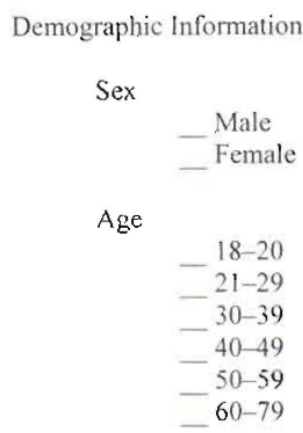

Highest Level of Education

High School
BA/BS
MA/MS
$\mathrm{PhD}$

On a scale of one to five - with I being "not at all familiar" and 5 being "very familiar" - how familiar are you with using touch screens to interact with a computer or phone?

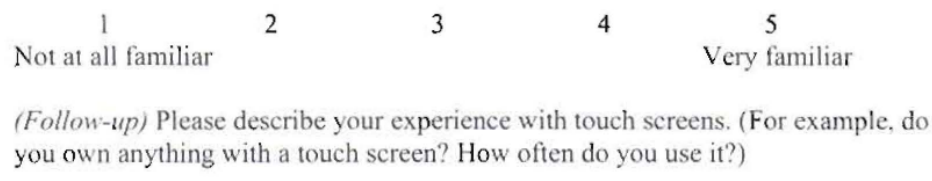




\section{Appendix 3, Exit Survey}

Exit Survey Questions

1. Was there a task you found especially difficult or easy? If so, why?

2. Were there parts of the interface you found confusing, or especially easy to understand? If so, why?

3. Were there parts of the interface that reacted in a way that was surprising, or predictable? If so, why?

4. (Optional, depending on time) Is there anything that you would like to change about the interface? 
Appendix 4, Study 1 Script, Operator Tasks First

Operator task 1st

\section{Informed consent}

a. any time an organization conducts research involving human participants, have to go through something called the informed consent process

b. making sure you are informed and aware your participation is $100 \%$ voluntary and you are free to withdraw at any time

c. Study should take roughly 30 minutes to complete; will go over the details shortly, but first I want to go over a few more parts of the informed consent process

d. 2 things I really want to emphasize: data will be completely anonymous and private

i. data identified only by number, not by name

ii. not collecting any personally identifiable information, or PII

iii. none of your biometric data recorded

e. data recording

i. I will be taking notes throughout the study, supplemented by video and audio recording.

ii. We are not recording yet, I will let you know before we start.

iii. Want to stress that the camera will be focused only on your hands and the device you'll be working with, not your face.

iv. Will be recording from the camera over your left shoulder; if you happen to look up into the camera during recording, we will delete any frames with a face image.

f. give you a chance to read and sign this, ask any questions you may have, then we'll be done with the informed consent process and start talking about the study itself.

g. Would you like a copy of the informed consent form?

\section{Demographic questionnaire}

\section{Study overview/purpose}

a. Today, you'll be using this device to help us test a new user interface for recording biometrics. Again, we're not recording any of your biometrics; we simply want to know what you think about using the interface. We're not testing you, we're testing the user interface. Your feedback today will be extremely valuable in helping improve it, so please share any and all comments and suggestions.

b. Have you used a device like this before? Not necessarily an iPad, could be something like an Android smartphone for example.

i. [if not, show iPad video clip]

\section{Explanation of special terms}

a. Before we get started, I need to explain $\mathbf{3}$ main terms: biometric, sensor, and workflow. Although you may already be familiar with these concepts, I still need to review them with you in order to be consistent across participants. 
b. Biometric: I'll be using the word "biometric" or "biometrics" quite frequently today. When I say "biometric," I'm simply referring to information about a person, such as a fingerprint, face image, iris image, voice pattern, or gait.

c. Sensor: sensors record the biometrics. Today we will only be using two sensors: [point to each sensor]

i. The first sensor is the webcam. This sensor records face images. I will refer to this as the webcam.

ii. The second sensor is the CrossMatch fingerprint scanner. This sensor records fingerprints. I will refer to this as the CrossMatch scanner or the CrossMatch sensor.

1. There are several different types of fingerprints: [demo these on sensor] a. Flat

b. Rolled

c. Both thumbs

d. Single finger

d. Workflow

e. All four fingers at once: this is called a slap

i. In the context of today's study, workflow refers to a series of biometrics recorded from specific sensors in a particular order. A short workflow might consist of Fingerprint from scanner A followed by face image from scanner B.

e. Questions on any of those terms, biometric, sensor, slap, workflow? 
Operator task 1 st

\section{Tasks groupings and talk-aloud}

a. The iPad interface controls these two sensors. For today, the focus is only on the iPad; don't worry about how the sensors work.

b. Now l'll describe a scenario for you and ask you to complete several tasks with the device. The tasks will be split into two groups.

c. As you work through each task, it would help a lot if you could talk aloud while you use the interface.

d. Here is the scenario for the first group of tasks:

\section{1st group of tasks (operator role)}

i. In this scenario, you will be playing the role of an operator responsible for taking all employees' biometrics. In your big, spacious, corner office, you have the CrossMatch fingerprint scanner and the webcam. You will be gathering data from two different employees, named Alice and Bob. I will be playing the role of both employees, and you will record my biometrics. You will be playing the role of the operator.

[turn on ipad and give to ptpt: model the angle they should hold it at, explain the tape]

1. I'm your new employee $\underline{\text { Alice, }}$, and I'm here in your office to have my left slap print collected.

a. TASK: Use the CrossMatch scanner to collect my left slap. As you complete the task, please remember to think aloud. If the interface does something you don't expect, etc.

b. TASK: The first result you get is unsatisfactory; what would you do now?

2. Now I need my picture taken.

a. TASK: Use the webcam to collect my face image.

3. Now I need my right slap print collected.

a. TASK: Use the CrossMatch scanner to collect my right slap.

b. ${ }^{* \star *}$ INTERRUPTION: I would like you to take a new picture since I wasn't smiling in the last one. SUBTASK: Return to the Face capture step, retake the picture, then return to where you were. [i.e., return to taking right slap]

4. I'm leaving! After I leave, you remember I was wearing a bandaid on my left index finger. Since I have gone for the day and you cannot retake this 
Operator task 1 st

print, annotate the left slap to indicate the index fingerprint could not be acquired.

5. Now I'm a different employee, named Bob. I'm in your office to have my biometrics recorded.

a. TASK: Use the CrossMatch scanner to collect my left slap.

b. TASK: Use the webcam to collect my face image.

c. TASK: Use the Crossmatch scanner to collect my right slap.

i. SUBTASK: During the collection of my right slap, I tell you Bob's not feeling well and can't continue. What would you do?

- Suppose you've taken a bunch of results, and it's now time to look at them in more detail

- TASK: Browse the results you've taken.

- Open the left slap image.

- Zoom in on the left slap image to see it larger.

- Browse to the next captured image.

- Return to the previous image.

- Return to the main sensor interface.

- You've completed that set of tasks, so let's move on to the next set. 
Operator task 1 st

\section{2nd group of tasks (manager role)}

e. Here is the scenario for the second group of tasks: [start with basic main screen, want them to find the "Create new workflow" button themselves]

i. In this scenario, you will be playing the role of the manager, setting the system up so others can collect the data you need.

- Now you're going to set up the system to take a series of biometrics in a row.

- TASK: Make a new workflow

- TASK: Set up this workflow so that it uses (in this order):

1. The Crossmatch sensor to capture a left slap

2. The Crossmatch sensor to capture a right slap

3. The webcam sensor to capture a face image.

- TASK: Rearrange the steps inside the workflow

- [if they drag newest to bottom] so that the face image is captured before the right slap.

- [if they drag newest to top] left slap is above face (or face below left slap)

- TASK: l'd like you to call this workflow Test \#_ [insert their ptpt \# here] Alternative instructions if "call" doesn't work: Name the workflow. Rename the workflow. Please title this workflow. Give it a name.

- Are you finished? [want them to find the Done button]

You've completed all the tasks, now I have a few short questions to ask you.

[administer Exit Survey] 
Appendix 5, Study 1 Script, Manager Tasks First

Manager task 1st

\section{Informed consent}

a. showing you understand what's being asked of you, can quit at any time

b. making sure you are informed and aware your participation is $100 \%$ voluntary and you are free to withdraw at any time

c. Study should take roughly 30 minutes to complete; will go over the details shortly, but first I want to go over a few more parts of the informed consent process

d. 2 things I really want to emphasize: data will be completely anonymous and private

i. data identified only by number, not by name

ii. not collecting any personally identifiable information, or PII

iii. none of your biometric data recorded

e. data recording

i. I will be taking notes throughout the study, supplemented by video and audio recording.

ii. We are not recording yet, I will let you know before we start.

iii. Want to stress that the camera will be focused only on your hands and the device you'll be working with, not your face.

iv. Will be recording from the camera over your left shoulder; if you happen to look up into the camera during recording, we will delete any frames with a face image.

f. give you a chance to read and sign this, ask any questions you may have, then we'll be done with the informed consent process and start talking about the study itself.

g. Would you like a copy of the informed consent form?

\section{Demographic questionnaire}

\section{Study overview/purpose}

a. Today, you'll be using this device to help us test a new user interface for recording biometrics. Again, we're not recording any of your biometrics; we simply want to know what you think about using the interface. We're not testing you, we're testing the user interface. Your feedback today will be extremely valuable in helping improve it, so please share any and all comments and suggestions.

b. Have you used a device like this before? Not necessarily an iPad, could be something like an Android smartphone for example.

i. [if not, show iPad video clip]

\section{Explanation of special terms}

a. Before we get started, I need to explain $\mathbf{3}$ main terms: biometric, sensor, and workflow. Although you may already be familiar with these concepts, I still need to review them with you in order to be consistent across participants. 
Manager task 1st

b. Biometric: I'll be using the word "biometric" or "biometrics" quite frequently today. When I say "biometric," I'm simply referring to information about a person, such as a fingerprint, face image, iris image, voice pattern, or gait.

c. Sensor: sensors record the biometrics. Today we will only be using two sensors: [point to each sensor]

i. The first sensor is the webcam. This sensor records face images. I will refer to this as the webcam.

ii. The second sensor is the CrossMatch fingerprint scanner. This sensor records fingerprints. I will refer to this as the CrossMatch scanner or the CrossMatch sensor.

1. There are several different types of fingerprints: [demo these on sensor] a. Flat

b. Rolled

c. Both thumbs

d. Single finger

e. All four fingers at once: this is called a slap

\section{d. Workflow}

i. In the context of today's study, workflow refers to a series of biometrics recorded from specific sensors in a particular order. A short workflow might consist of Fingerprint from scanner A followed by face image from scanner B.

e. Questions on any of those terms, biometric, sensor, slap, workflow? 
Manager task 1st

\section{Tasks groupings and talk-aloud}

a. The iPad interface controls these two sensors. For today, the focus is only on the iPad; don't worry about how the sensors work.

b. Now l'll describe a scenario for you and ask you to complete several tasks with the device. The tasks will be split into two groups.

c. As you work through each task, it would help a lot if you could talk aloud while you use the interface.

d. Here is the scenario for the first group of tasks: [start with basic main screen, want them to find the "Create new workflow" button themselves]

\section{1st group of tasks (manager role)}

i. In this scenario, you will be playing the role of the manager, setting the system up so others can collect the data you need.

[turn on ipad and give to ptpt: model the angle they should hold it at, explain the tape]

- Now you're going to set up the system to take a series of biometrics in a row. As you complete the task, please remember to talk aloud. If the interface does something you don't expect, etc.

- TASK: Make a new workflow

- TASK: Set up this workflow so that it uses (in this order):

1. The Crossmatch sensor to capture a left slap

2. The Crossmatch sensor to capture a right slap

3. The webcam sensor to capture a face image.

- TASK: Rearrange the steps inside the workflow

- [if they drag newest to bottom-default behavior if they use the add new button] so that the face image is captured before the right slap.

- [if they drag newest to top] left slap is captured before the face (or face after left slap)

- TASK: l'd like you to call this workflow Test \#_ [insert their ptpt \# here] Alternative instructions if "call" doesn't work: Name the workflow. Rename the workflow. Please title this workflow. Give it a name.

- Are you finished? [want them to find the Done button]

- You've completed that set of tasks, so let's move on to the next set. 
Manager task 1st

\section{2nd group of tasks (operator role)}

e. Here is the scenario for the second group of tasks:

i. In this scenario, you will be playing the role of an operator responsible for taking all employees' biometrics. In your big, spacious, corner office, you have the CrossMatch fingerprint scanner and the webcam. The iPad interface controls these two sensors. For today, the focus is only on the iPad; don't worry about how the sensors work. You will be gathering data from two different employees, named Alice and Bob. I will be playing the role of both employees, and you will record my biometrics. You will be playing the role of the operator.

[load pre-made workflow for them, "User Testing-Operator \#]

1. I'm your new employee $\underline{\text { Alice, }}$, and I'm here in your office to have my left slap print collected.

a. TASK: Use the CrossMatch scanner to collect my left slap. As you complete the task, please remember to think aloud. If the interface does something you don't expect, etc.

b. TASK: The first result you get is unsatisfactory; what would you do now?

2. Now I need my picture taken.

a. TASK: Use the webcam to collect my face image.

3. Now I need my right slap print collected.

a. TASK: Use the CrossMatch scanner to collect my right slap.

b. ***INTERRUPTION: I would like you to take a new picture since I wasn't smiling in the last one. SUBTASK: Return to the Face capture step, retake the picture, then return to where you were. [i.e., return to taking right slap]

4. I'm leaving! After I leave, you remember I was wearing a bandaid on my left index finger. Since I have gone for the day and you cannot retake this print, mark the left slap to indicate the index fingerprint could not be acquired.

5. Now I'm a different employee, named Bob. I'm in your office to have my biometrics recorded.

a. TASK: Use the CrossMatch scanner to collect my left slap.

b. TASK: Use the webcam to collect my face image.

c. TASK: Use the Crossmatch scanner to collect my right slap.

i. [don't actually put right hand on platten]

ii. SUBTASK: During the collection of my right slap, I tell you Bob's not feeling well and can't continue. What would you do? 


\title{
Appendix 6, Study 2 Script
}

\author{
wsabi 2.0 script
}

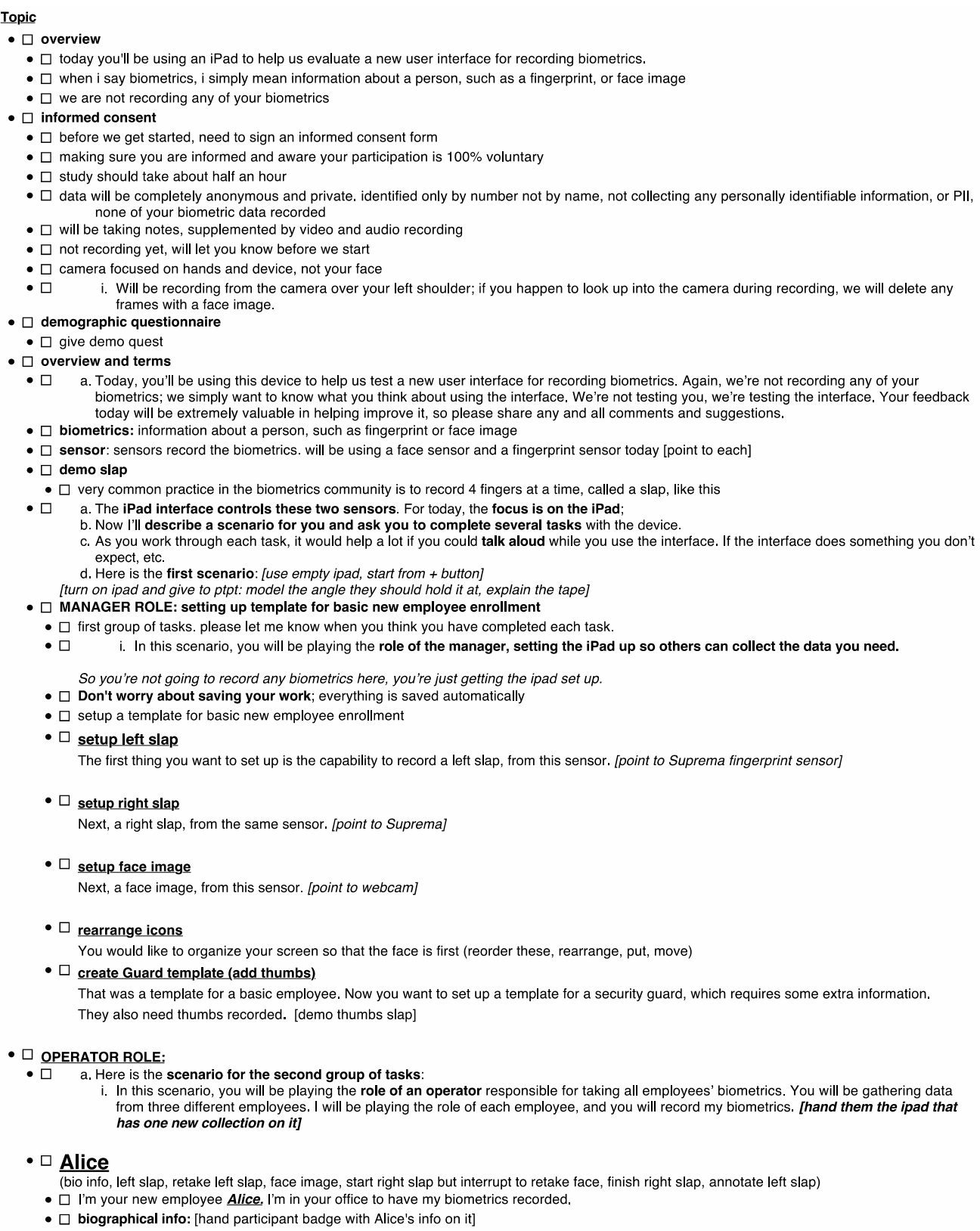


This publication is available free of charge from http://dx.doi.org/10.6028/NIST.IR.8003

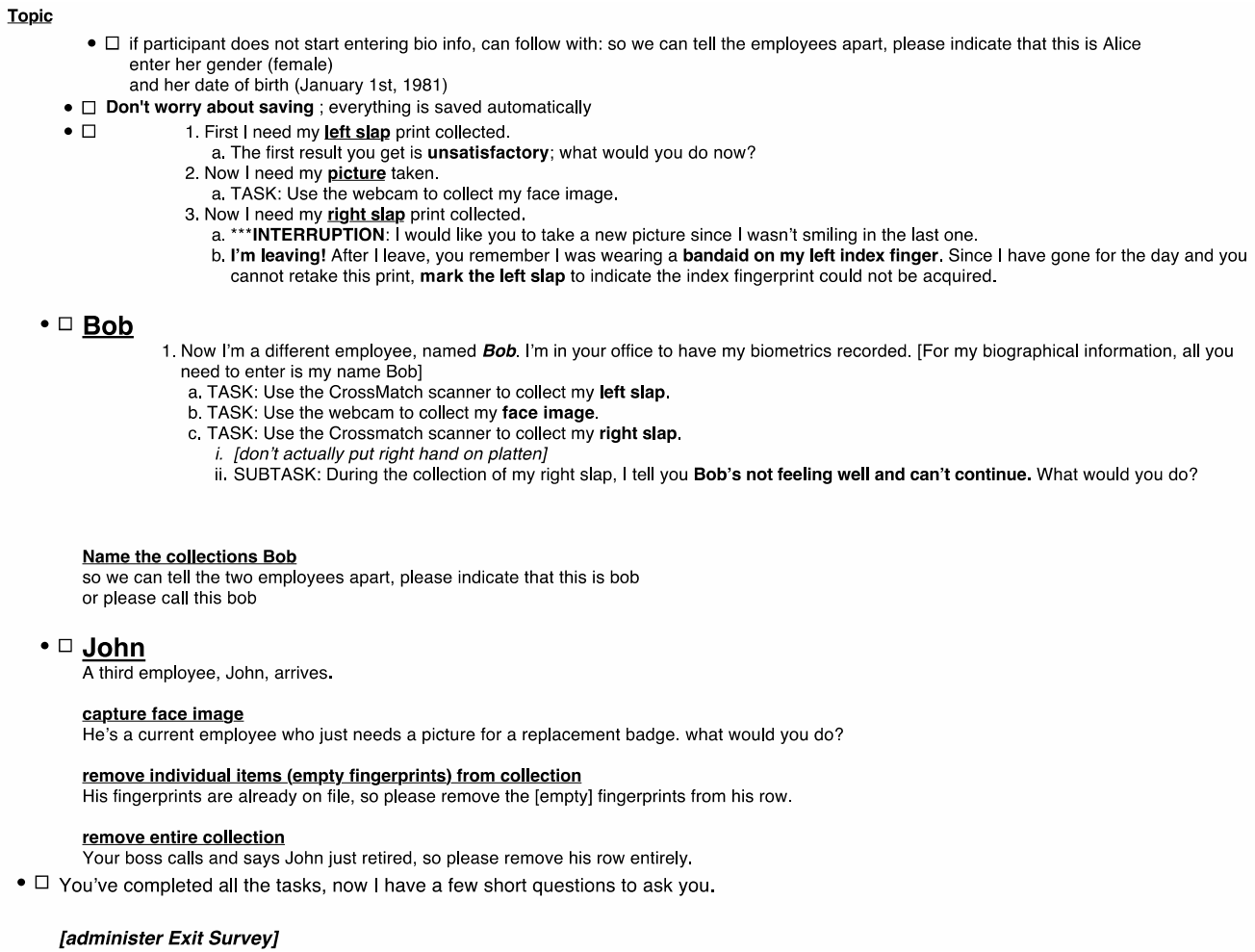

